\title{
Enhanced sampling schemes for MCMC based blind Bernoulli-Gaussian deconvolution
}

\author{
D. Ge ${ }^{\mathrm{a}, *}$ J. Idier ${ }^{\mathrm{a}}$ E. Le Carpentier ${ }^{\mathrm{a}}$ \\ ${ }^{\mathrm{a}}$ IRCCyN (CNRS UMR 6597), 1 rue de la Noë, BP 92101, 44321 Nantes Cedex 3, France
}

\begin{abstract}
This paper proposes and compares two new sampling schemes for sparse deconvolution using a Bernoulli-Gaussian model. To tackle such a deconvolution problem in a blind and unsupervised context, the Markov Chain Monte Carlo (MCMC) framework is usually adopted, and the chosen sampling scheme is most often the Gibbs sampler. However, such a sampling scheme fails to explore the state space efficiently. Our first alternative, the $K$-tuple Gibbs sampler, is simply a grouped Gibbs sampler. The second one, called partially marginalized sampler, is obtained by integrating the Gaussian amplitudes out of the target distribution. While the mathematical validity of the first scheme is obvious as a particular instance of the Gibbs sampler, a more detailed analysis is provided to prove the validity of the second scheme.

For both methods, optimized implementations are proposed in terms of computation and storage cost. Finally, simulation results validate both schemes as more efficient in terms of convergence time compared with the plain Gibbs sampler. Benchmark sequence simulations show that the partially marginalized sampler takes fewer iterations to converge than the $K$-tuple Gibbs sampler. However, its computation load per iteration grows almost quadratically with respect to the data length, while it only grows linearly for the $K$-tuple Gibbs sampler.
\end{abstract}

Key words: Blind deconvolution, Bernoulli-Gaussian model, Markov chain Monte Carlo methods

\footnotetext{
* Corresponding author. Tel.:+332403769 24; fax: +33240376930

Email addresses: Di.Ge@irccyn.ec-nantes.fr (D. Ge), Jerome.Idier@irccyn.ec-nantes.fr (J. Idier), Eric.Le-Carpentier@irccyn.ec-nantes.fr (E. Le Carpentier).
} 


\section{Introduction}

The problem of the restoration of a sparse spike train $\boldsymbol{x}$ distorted by a linear system $\boldsymbol{h}$ and corrupted by noise $\epsilon$ such that $\boldsymbol{z}=\boldsymbol{x} * \boldsymbol{h}+\epsilon$, arises in many fields such as seismic exploration [12] and astronomy [3].

In this paper, we adopt a Bernoulli-Gaussian (BG) model for the spike train $\boldsymbol{x}$, following [2] and many subsequent contributions such as [1]4. A BG signal is an independent, identically distributed (iid) process defined in two stages. Firstly, the sparse nature of the spikes is governed by the Bernoulli law:

$$
P(\boldsymbol{q})=\lambda^{L}(1-\lambda)^{M-L}
$$

with the Bernoulli sequence $\boldsymbol{q}=\left[q_{1}, \ldots, q_{M}\right]^{\mathrm{t}}$ and $L=\sum_{m=1}^{M} q_{m}$, the number of non-zero realizations. Secondly, amplitudes $\boldsymbol{x}=\left[x_{1}, \ldots, x_{M}\right]^{\mathrm{t}}$ are assumed iid zero-mean Gaussian conditionally to $\boldsymbol{q}$ :

$$
\boldsymbol{x} \mid \boldsymbol{q} \sim \mathcal{N}\left(\mathbf{0}, \sigma_{x}^{2} \operatorname{diag}(\boldsymbol{q})\right),
$$

where $\operatorname{diag}(\boldsymbol{q})$ denotes a diagonal matrix whose diagonal is $\boldsymbol{q}$.

The MCMC approach [5]6] is a powerful numerical tool, appropriate to solve complex inference problems such as blind deconvolution. In the field of blind BG deconvolution, Cheng et al. pioneered the introduction of MCMC methods [1. They proposed to rely on a plain Gibbs sampler, i.e., with a site-by-site updating scheme for the spike train, for which their algorithm constitutes a simple and canonical example. However, simulation results indicate that it lacks reliability: from different initial conditions, significantly different estimations are obtained, even after a considerable number of iterations.

The recent contribution of [7] already identified a convergence issue linked to time-shift ambiguities, and proposed an efficient way to solve it. In addition, the scale ambiguities are treated by [ $[8]$ by proposing a scale re-sampling step to accelerate the convergence rate of the Markov chain. In this study, we point out another source of inefficiency, unrelated to the above-mentioned ambiguities: instead of exploring the $2^{M}$ configurations of $\boldsymbol{q}$ at an acceptable speed, the Gibbs sampler tends to get stuck for many iterations around some particular configurations of $\boldsymbol{q}$, often corresponding to local optimal configurations of $\boldsymbol{x}$ of the posterior distribution as illustrated by the example in Section 2.2. This conclusion meets Bourguignon and Carfantan's analysis [3]: the Markov chain equilibrates rapidly around a mode (i.e., a local optimal configuration), but takes a long time to move from mode to mode.

In order to make up for this deficiency, our first proposition is to adopt a grouped Gibbs sampler [5], called a $K$-tuple Gibbs sampler, where blocks of $K$ adjacent BG variables $\left(q_{i}, \ldots, q_{i+K-1}\right)$ and their associated 
amplitudes $\left(x_{i}, \ldots, x_{i+K-1}\right)$ are jointly sampled. A comparable idea first appeared in [9], and more recently in [3], under the form of a deterministic iteration aimed at producing an increase of the posterior probability, whereas our goal is to sample the latter.

We then propose a second solution based on sampling the posterior distribution marginally to the amplitudes $\boldsymbol{x}$ [10]. A comparable idea is found in statistical signal segmentation [11] where some hyper-parameters are partially marginalized. In fact, as Liu pointed out in [5], completely integrating out some components (the Gaussian amplitudes $\boldsymbol{x}$ in our case), leads to a more efficient sampling scheme called collapsed Gibbs sampling. However, a plain collapsed Gibbs sampling on the marginal posterior distribution involves hardly tractable sampling steps. In particular, it is all but simple to sample $\boldsymbol{h}$ conditional on $(\boldsymbol{z}, \boldsymbol{q})$ and marginally with respect to $\boldsymbol{x}$. Our scheme solves this problem by combining a step that samples $\boldsymbol{q}$ marginally with respect to $\boldsymbol{x}$ and other sampling steps involving $\boldsymbol{x}$. Such a partially marginalized sampler is fully valid from the mathematical point of view. In this paper, we show that it can be interpreted as a plain Gibbs sampler with a particular scanning order of the variables.

Simulation tests on toy examples and on the so-called Mendel's sequence [2] confirm the efficiency of both methods in terms of computation time before convergence of the Markov chain. Further analysis shows the data length as a criterion to choose between the two proposed methods.

This paper is organized as follows. In Section 2, after a brief formulation of the blind BG deconvolution problem, the Gibbs sampler of the joint posterior distribution [1] is presented, and an example illustrates its inefficiency as regards the sampling of $\boldsymbol{q}$. Sections 3 and 4 respectively introduce the generalized $K$-tuple Gibbs sampler and the partially marginalized sampler. In both cases, a toy example is used to evaluate the capability of the sampler to escape from local optimal configurations, and implementation issues are carefully dealt with.

Finally, simulation results are presented in Section $[$ to compare the efficiency of the sampling schemes according to Brooks and Gelman's convergence diagnostic [12], and conclusions are drawn in Section [6.

\section{Problem formulation}

\subsection{Statistical model}

The mathematical model of convolution reads

$$
z_{n}=\sum_{k=0}^{P} h_{k} x_{n-k}+\epsilon_{n}
$$


for all $n \in\{1, \ldots, N\}$, where $\boldsymbol{z}=\left[z_{1}, \ldots, z_{N}\right]^{\mathrm{t}}$ denotes the observed vector, $\boldsymbol{x}=\left[x_{1}, \ldots, x_{M}\right]^{\mathrm{t}}$ is the unknown spike train, $\boldsymbol{h}=\left[h_{0}, \ldots, h_{P}\right]^{\mathrm{t}}$ the impulse response (IR) of the system (assumed finite here) and $\boldsymbol{\epsilon}=\left[\epsilon_{1}, \ldots, \epsilon_{M}\right]^{\mathrm{t}}$ an noise vector, often assumed white stationary Gaussian. The deconvolution problem is said blind when $\boldsymbol{h}$ is unknown, which is the studied case here. Akin to [1], the following assumptions are made:

$-\boldsymbol{\epsilon} \sim \mathcal{N}\left(0, \sigma_{\epsilon}^{2} \mathbf{I}\right)$ is independent of $\boldsymbol{x}$ and $\boldsymbol{h} ;$

- $\boldsymbol{x}$ is a BG process defined by Eqs.(1)-(2) with $\sigma_{\boldsymbol{x}}^{2}=1$;

$-\boldsymbol{h} \sim \mathcal{N}\left(0, \sigma_{h}^{2} \mathbf{I}_{P+1}\right)$.

Let us remark that by setting $\sigma_{\boldsymbol{x}}^{2}$ arbitrarily to one while imposing the Gaussian prior law $\boldsymbol{h}$ removes the scale ambiguity inherent to the blind deconvolution problem [7]. By adopting the "zero boundary" condition so that $M=N-P, \boldsymbol{z}$ can be rewritten in the form of a matrix multiplication $\boldsymbol{z}=\mathbf{H} \boldsymbol{x}+\boldsymbol{\epsilon}$, where $\mathbf{H}$ denote the $N \times M$ Toeplitz matrix of convolution. It is also useful to introduce the Toeplitz matrix $\mathbf{X}$ of size $N \times(P+1)$ such that $\mathbf{H} \boldsymbol{x}=\mathbf{X} \boldsymbol{h}$. According to the Monte Carlo principle, a posterior mean estimator of $\Theta=\left\{\boldsymbol{q}, \boldsymbol{x}, \boldsymbol{h}, \lambda, \sigma_{\epsilon}^{2}, \sigma_{\boldsymbol{h}}^{2}\right\}$ given $\boldsymbol{z}$ can be approximated by:

$$
\widehat{\Theta}=\frac{1}{I-J} \sum_{k=J+1}^{I} \Theta^{(k)},
$$

where the sum extends over the last $I-J$ samples. In the MCMC framework, the samples are generated iteratively, so that asymptotically $\Theta^{(k)}$ follows the joint posterior distribution [6]:

$$
P(\Theta \mid \boldsymbol{z}) \propto g\left(\boldsymbol{z}-\mathbf{H} \boldsymbol{x} ; \sigma_{\epsilon}^{2} \mathbf{I}_{N}\right) g\left(\boldsymbol{x} ; \sigma_{\boldsymbol{x}}^{2} \operatorname{diag}\{\boldsymbol{q}\}\right) g\left(\boldsymbol{h} ; \sigma_{h}^{2} \mathbf{I}_{P+1}\right) P(\boldsymbol{q} ; \lambda) P\left(\sigma_{\boldsymbol{h}}^{2}\right) P(\lambda) P\left(\sigma_{\epsilon}^{2}\right)
$$

where $g(\cdot ; \mathbf{R})$ denotes the centered Gaussian density of covariance $\mathbf{R}$. Conjugate prior laws are adopted for the last three terms $P\left(\sigma_{\boldsymbol{h}}^{2}\right), P(\lambda)$ and $P\left(\sigma_{\epsilon}^{2}\right)$ :

$$
\sigma_{\boldsymbol{h}}^{2} \sim I G(1,1), \quad \lambda \sim B e(1,1), \quad \sigma_{\epsilon}^{2} \sim I G(1,1)
$$

where $I G$ and $B e$ respectively represent the inverse Gamma and Beta distributions. The flat shapes of the two distributions $I G(1,1)$ and $B e(1,1)$ (a uniform distribution on $[0,1]$ ) can be interpreted as conveying no specific prior information of the parameters.

\subsection{Classical Gibbs Sampling}

A Gibbs sampler circumvents the difficulties of directly inferencing from the joint posterior distribution as in Eq. (5) and instead draws each parameter from its conditional law while all others are fixed. The principle 
of Cheng et al.'s Gibbs sampler is given in Table 1 and a pseudocode can be found in Table 2, The five simulation steps are iterated until convergence toward the posterior distribution in Eq. (5), and $\widehat{\Theta}$ is finally built according to (4). Let us remark than in Table 2, Step 1 is performed in two substeps: for each $i, q_{i}$ is first sampled conditional on $\left(\Theta \backslash\left(q_{i}, x_{i}\right), \boldsymbol{z}\right)$, and then $x_{i}$ conditional on $\left(\Theta \backslash x_{i}, \boldsymbol{z}\right)$. Taken jointly, these two substeps perform the sampling of $y_{i}=\left(q_{i}, x_{i}\right)$ conditional on $\left(\Theta \backslash y_{i}, \boldsymbol{z}\right)$.

Table 1

Cheng et al.'s Gibbs sampler.

(1) Let $\boldsymbol{y}=(\boldsymbol{q}, \boldsymbol{x})$. For each $i=1 \ldots, M$, draw $y_{i}^{(k+1)} \mid \boldsymbol{y}_{1: i-1}^{(k+1)}, \boldsymbol{y}_{i+1: M}^{(k)}, \boldsymbol{h}^{(k)}, \sigma_{\epsilon}^{(k)}, \lambda^{(k)}, \boldsymbol{z}$

(2) draw $\boldsymbol{h}^{(k+1)} \mid \boldsymbol{x}^{(k+1)}, \sigma_{\boldsymbol{h}}^{(k)}, \sigma_{\epsilon}^{(k)}, \boldsymbol{z}$

(3) draw $\sigma_{\epsilon}^{(k+1)} \mid \boldsymbol{x}^{(k+1)}, \boldsymbol{h}^{(k+1)}, \boldsymbol{z}$

(4) draw $\lambda^{(k+1)} \mid \boldsymbol{q}^{(k+1)}$

(5) draw $\sigma_{\boldsymbol{h}}^{(k+1)} \mid \boldsymbol{h}^{(k+1)}$

Table 2

A pseudocode for Cheng et al.'s Gibbs sampler (see 1 for implementation details).

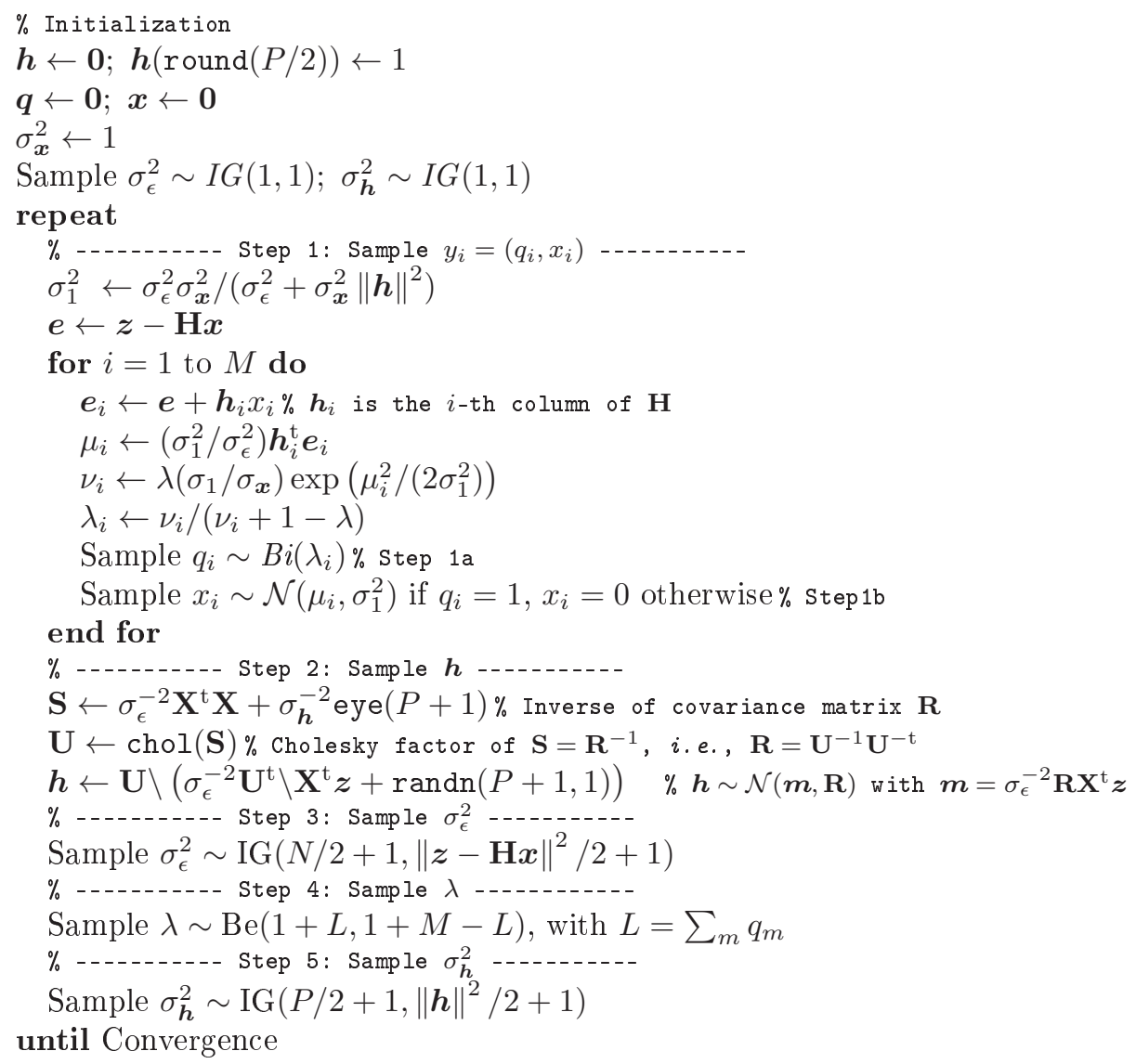


Despite its simplicity, this scheme presents several drawbacks. In [7], it is pointed out that time-shift ambiguities may lead to unreliable estimates, depending on the initialization of $\boldsymbol{h}$. To make up for this deficiency, an additional Metropolis-Hasting (MH) step is proposed between Steps 1 and 2 in order to allow moves to a time-shifted version of $(\boldsymbol{y}, \boldsymbol{h})$. Another inefficiency in the Cheng et al.'s Gibbs sampler has been discussed in [8] with regard to the scale factor between $\boldsymbol{h}$ and $\boldsymbol{x}$. A scale re-sampling step is proposed and tested to yield faster convergence. In Table 3 , the modified version of Step 2 incorporates the timeshift operation and the scale re-sampling with an efficient implementation in which $f_{\text {GIG }}$ represents the Generalized Inverse Gaussian law (see [7] and [8] for more details). In what follows, the resulting global scheme is referred to as a hybrid sampler, since there is a Metropolis Hastings step within the Gibbs scheme and no more a pure Gibbs sampler.

Table 3

Modified version of Step 2 to incorporate the time-shift compensation according to [7] and the scale re-sampling according to 8 .

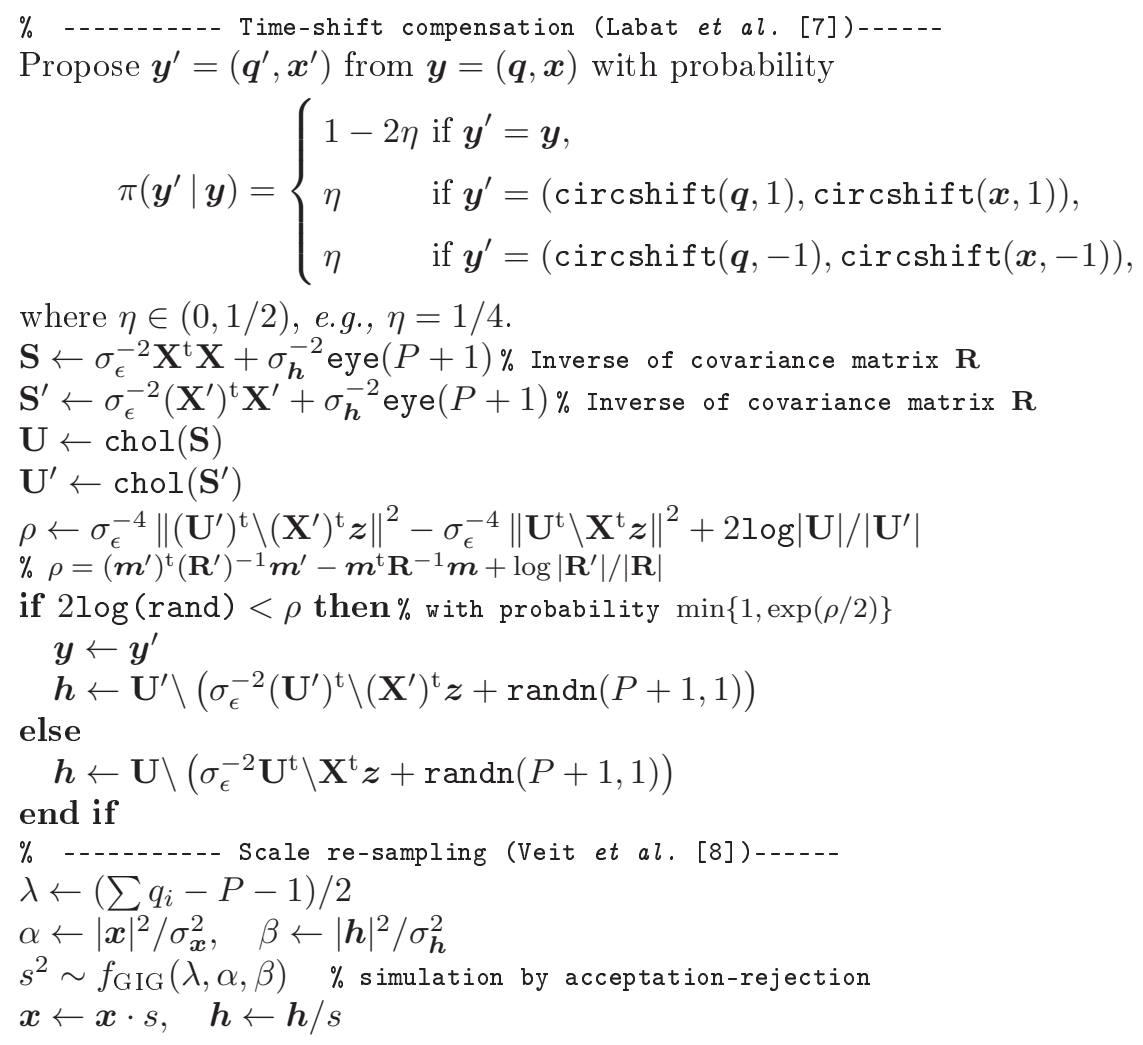

Despite the improvement brought by the timeshift and scale ressampling operations in the hybrid sampler, we have identified another source of inefficiency, completely independent from the time-shift and/or scale ambiguity problem. Figure 1 is an illustration based on simulated data generated from a single spike, 
convolved with an IR defined by

$$
\boldsymbol{h}(i)=\cos \left((i-10) \frac{\pi}{4}\right) \exp \left(-|0.225 i-2|^{1.5}\right), i=0, \ldots, 20,
$$

which is depicted by bullets on Figure 2(b). A local optimal configuration of $\boldsymbol{x}$ (two neighboring spikes instead of a single one) is chosen as the initial state in Figure1(a). The hybrid sampler generates a Markov chain that typically takes several thousands of iterations (depending on the chosen random generator seed) to visit the optimal configuration for the first time. Figure2 further illustrates that the hybrid sampler tends to

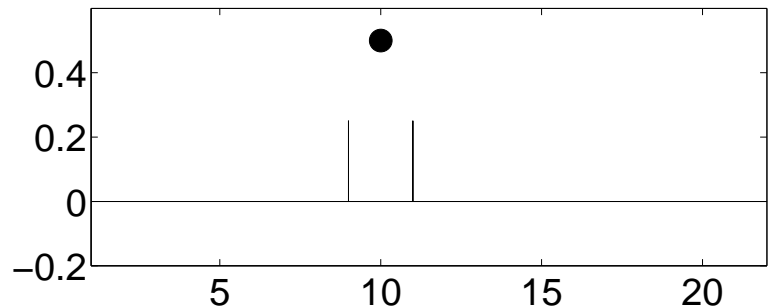

(a) Initial configuration.

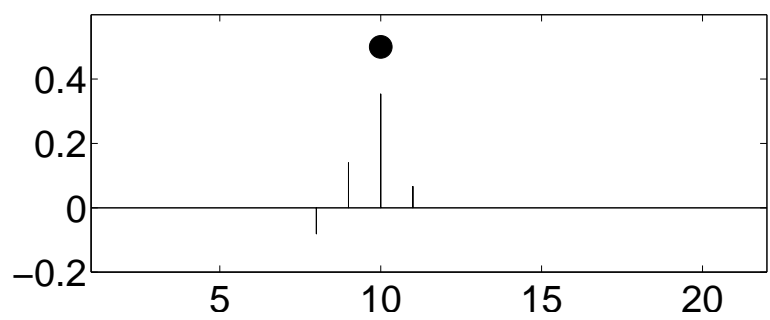

(c) 2000th iteration

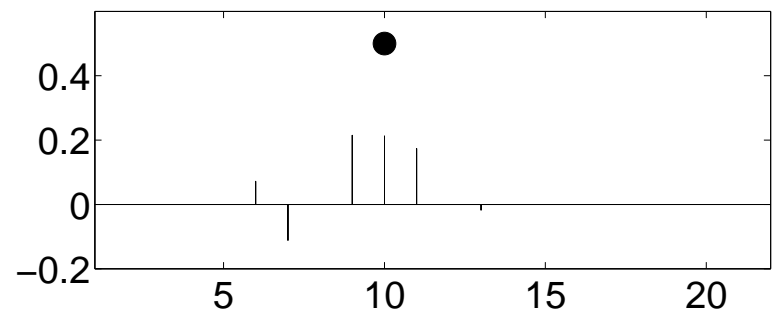

(b) 1000th iteration

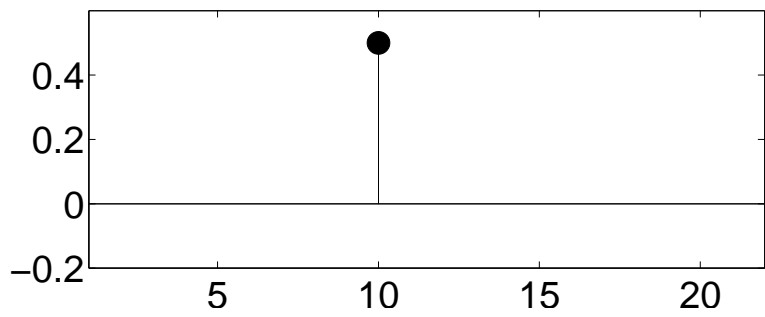

(d) 2415 th iteration

Fig. 1. Sampled BG sequences obtained by the hybrid sampler on a simple example. From a local optimal configuration chosen as initial state, the Markov chain spends several hundreds of iterations before visiting the solution, i.e., a unique spike in position 10 (marked as a bullet).

produce unreliable estimated values. Here, Mendel's well-known BG sequence is adopted as a test signal [2]. The same IR is used and the data are corrupted by Gaussian noise with $\sigma_{\epsilon}^{2}=4 \times 10^{-6}$, corresponding to a signal-to-noise ratio (SNR) of $12.80 \mathrm{~dB}$. The three estimation results in Figure 2 are obtained from the same simulated data $\boldsymbol{z}$, and the same initial states, the only difference being the seed value of the random generator. For each Markov chain, 1000 samples are produced and the last 250 are averaged to compute the estimation. Substantial variations exist from one estimated result to another, especially in the number and the positions of spikes in Figure2(a). Actually, it can be checked that each sequence $\left\{\boldsymbol{q}^{(k)}\right\}$ tends to remain constant for many iterations, instead of exploring the state space efficiently. It should be stressed that these results are typical, i.e., they have not been selected on purpose.

This phenomenon is basically due to Step 1 of the sampler. More precisely, the corresponding sampled 

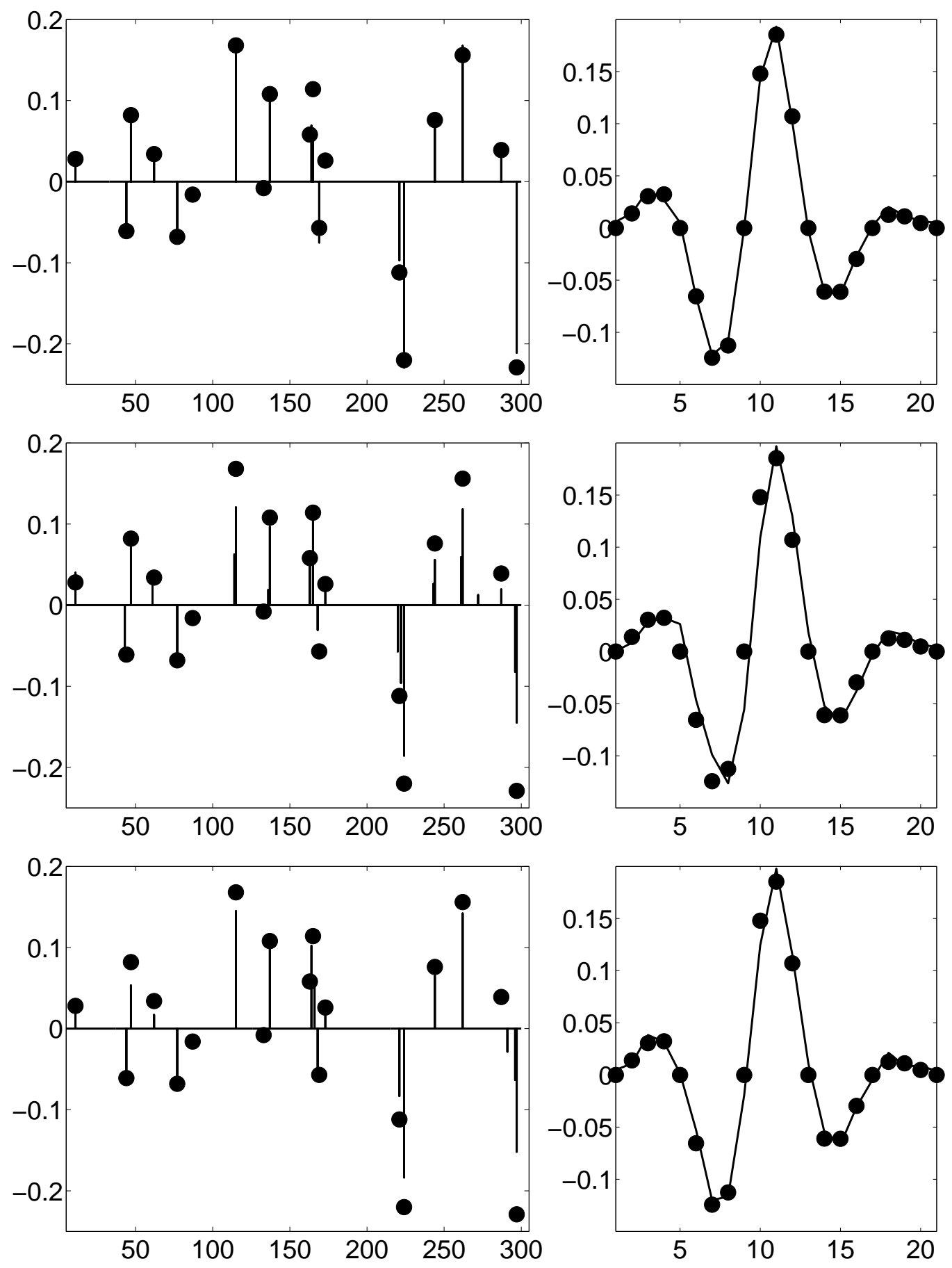

(a) estimation of $\boldsymbol{x}$

(b) estimation of $\boldsymbol{h}$

Fig. 2. Three different solutions of the hybrid sampler [7, obtained by changing the random generator seed only. The actual values are marked as bullets.

chains hardly escape from local optima of the posterior distribution, because the consecutive samples are highly dependent. 


\section{Generalized Gibbs on K-tuple variables}

This inefficiency in MCMC sampling schemes has been already noticed by Bourguignon and Carfantan in [3. They propose a solution involving shifts of detected spikes to adjacent positions. However, such a solution is a deterministic procedure aimed at increasing the posterior probability during the burn-in period of the Markov chain. Therefore, it does not leave the posterior distribution invariant. In contrast, our goal here is to propose a valid sampling step that produces spike shifts.

Let us introduce the set $\Omega=\{0, \ldots, K-1\}$, and the notation $i+\omega=\{i+m \mid m \in \omega\}$ for all $\omega \subset \Omega$. For any vector $\boldsymbol{v}$, let us denote $\boldsymbol{v}_{\omega}$ the subvector formed by the entries $v_{m}, m \in \omega$, and $\boldsymbol{v}_{-\omega}$ the subvector formed by the remaining entries. Similarly, for any matrix $\boldsymbol{V}$, we introduce the submatrices $\boldsymbol{V}_{\omega}$ and $\boldsymbol{V}_{-\omega}$ formed by columns of $\boldsymbol{V}$, indexed by $\omega$ in the first case, and gathering the remaining columns in the second case.

The principle of the grouped Gibbs sampler to K-tuple variables consists in updating vector $\boldsymbol{y}_{i+\Omega}$ for each $i=1, \ldots, M-K+1$ instead of the scalar updates of Step 1 in Table 1 In particular, let us remark that such a joint update strategy allows a permutation of the Bernoulli vector $\boldsymbol{q}_{i+\Omega}$ (from a configuration $(0,1)$ to $(1,0)$ in the case of $K=2)$ within one iteration.

The resulting sampling scheme is given in Table 4. The notation of $\boldsymbol{q}_{i+\Omega}^{(k+1 / 2)}$ (and that of $\boldsymbol{x}_{i+\Omega}^{(k+1 / 2)}$ ) denotes the fact that $\boldsymbol{y}^{(k+1)}$ is latter re-sampled in step 2(a). Compared to the hybrid version of the original Gibbs sampler of Table 1, only Step 1 has been modified. It is clear that in the case of $K=1$, we are driven back to the hybrid sampler.

Table 4

Grouped Gibbs sampler updating $K$-tuple variables

(1) For each $i=1 \ldots, M-K+1$,

(a) draw $\boldsymbol{q}_{i+\Omega}^{(k+1 / 2)} \mid \boldsymbol{y}_{-(i+\Omega)}^{(k)}, \boldsymbol{h}^{(k)}, \sigma_{\epsilon}^{(k)}, \lambda^{(k)}, \boldsymbol{z}$

(b) draw $\boldsymbol{x}_{i+\Omega}^{(k+1 / 2)} \mid \boldsymbol{y}_{-(i+\Omega)}^{(k)}, \boldsymbol{q}_{i+\Omega}^{(k+1 / 2)}, \boldsymbol{h}^{(k)}, \sigma_{\epsilon}^{(k)}, \boldsymbol{z}$

(2) (a) draw $\boldsymbol{y}^{(k+1)}$ according to Table 3

(b) draw $\boldsymbol{h}^{(k+1)} \mid \boldsymbol{y}^{(k+1)}, \sigma_{\boldsymbol{h}}^{(k)}, \sigma_{\epsilon}^{(k)}, \boldsymbol{z}$

(3) draw $\sigma_{\epsilon}^{(k+1)} \mid \boldsymbol{x}^{(k+1)}, \boldsymbol{h}^{(k+1)}, \boldsymbol{z}$

(4) draw $\lambda^{(k+1)} \mid \boldsymbol{q}^{(k+1)}$

(5) draw $\sigma_{\boldsymbol{h}}^{(k+1)} \mid \boldsymbol{h}^{(k+1)}$

It should be noted that the complexity of the $K$-tuple sampler increases rapidly (indeed, exponentially) with $K$, while larger values of $K$ are expected to allow a more efficient exploration of the state space. 
The latter fact is illustrated by Table 5 , which reports the number of iterations needed to visit the exact configuration of $\boldsymbol{q}$ for the first time, for different values of $K$ and of the random generator seed. For $K=1$, the required number of iterations varies in large amounts. Typically, several thousands of iterations are required. In contrast, the exact configuration of $\boldsymbol{q}$ is visited after a few tens of iterations for $K=2$. For $K=3$ or $K=4$, the first time visit happens immediatly in most cases.

Table 5

Number of iterations for $K$-tuple samplers to visit the true configuration for the first time in the example of Figure 1

\begin{tabular}{|c|c|c|c|c|}
\hline seed value & $K=1$ & $K=2$ & $K=3$ & $K=4$ \\
\hline 19 & 2415 & 21 & 4 & 1 \\
\hline 29 & 1558 & 55 & 1 & 1 \\
\hline 39 & 5137 & 12 & 1 & 1 \\
\hline 49 & 7132 & 37 & 3 & 2 \\
\hline
\end{tabular}

In practice, the question remains to know what is the best trade-off between $K=1$ or larger values of $K$. In the first case, the convergence is slow in terms of iteration number, but the computing time for each iteration is relatively low. By increasing values of $K$, the computing time per iteration increases rapidly, while the iteration number decreases. Finding the best trade-off on a purely theoretical and general basis is probably hopeless. More modestly, we examine this question in Section 5 on the sole basis of Mendel's example of Figure 2. However, a prerequisite is to rely on an optimized implementation of the K-tuple algorithm. This is the goal of the next subsection.

\subsection{Numerical implementation}

Steps 2 to 5 being identical to those of the hybrid sampler, only the algorithmic implementation of the Step 1 is detailed in Table 6. In the K-tuple sampler, Step 1 draws $\left(\boldsymbol{q}_{i+\Omega}, \boldsymbol{x}_{i+\Omega}\right)$ according to their joint conditional law:

$$
\begin{aligned}
P\left(\boldsymbol{q}_{i+\Omega}, \boldsymbol{x}_{i+\Omega} \mid \text { rest }\right) & \propto P\left(\boldsymbol{z} \mid \boldsymbol{x}_{i+\Omega}, \boldsymbol{x}_{-(i+\Omega)}, \boldsymbol{h}, \sigma_{\epsilon}^{2}\right) P\left(\boldsymbol{x}_{i+\Omega} \mid \boldsymbol{q}_{i+\Omega}, \sigma_{x}^{2}\right) P\left(\boldsymbol{q}_{i+\Omega} \mid \lambda\right) \\
& \propto \exp \left\{-\frac{1}{2 \sigma_{\epsilon}^{2}}\left\|\boldsymbol{e}_{K, i}-\mathbf{H}_{i+\Omega} \boldsymbol{x}_{i+\Omega}\right\|^{2}\right\} g\left(\boldsymbol{x}_{i+\Omega} ; \sigma_{x}^{2} \operatorname{diag}\left(\boldsymbol{q}_{i+\Omega}\right)\right)\left(\frac{\lambda}{1-\lambda}\right)^{\mathbf{1}^{\dagger} \boldsymbol{q}_{i+\Omega}}
\end{aligned}
$$

where $\boldsymbol{e}_{K, i}=\boldsymbol{z}-\mathbf{H}_{-(i+\Omega)} \boldsymbol{x}_{-(i+\Omega)}$, so that $\boldsymbol{e}_{K, i}$ is a function of $\left(\boldsymbol{x}_{-(i+\Omega)}, \boldsymbol{h}, \boldsymbol{z}\right)$ but not of $\boldsymbol{x}_{i+\Omega}$.

Let $\omega \subset \Omega$ be such that $\boldsymbol{q}_{i+\omega}$ gathers the nonzero entries of $\boldsymbol{q}_{i+\Omega}$, so that $\mathbf{1}^{\mathrm{t}} \boldsymbol{q}_{i+\Omega}=\# \omega$. From (6), it is straightforward to deduce that Step 1(b) amounts to draw a Gaussian law defined by $\boldsymbol{x}_{i+\Omega \backslash \omega}=\mathbf{0}$ and, if $\omega \neq \emptyset, \boldsymbol{x}_{i+\omega} \sim \mathcal{N}\left(\boldsymbol{m}_{i+\omega}, \mathbf{R}_{i+\omega}\right)$, where 


$$
\begin{aligned}
& \mathbf{R}_{i+\omega}^{-1}=\sigma_{\epsilon}^{-2} \mathbf{H}_{i+\omega}^{\mathrm{t}} \mathbf{H}_{i+\omega}+\sigma_{\boldsymbol{x}}^{-2} \mathbf{I}_{\# \omega}, \\
& \boldsymbol{m}_{i+\omega}=\sigma_{\epsilon}^{-2} \mathbf{R}_{i+\omega} \mathbf{H}_{i+\omega}^{\mathrm{t}} \boldsymbol{e}_{K, i} .
\end{aligned}
$$

By integration of (6) with respect to $\boldsymbol{x}_{i+\Omega}$, it is then possible to express the marginal conditional law of $\boldsymbol{q}_{i+\Omega}$, along which the latter vector must be sampled according to Step 1(a):

$$
P\left(\boldsymbol{q}_{i+\omega}=\mathbf{1}, \boldsymbol{q}_{i+\Omega \backslash \omega}=\mathbf{0}\right)=\frac{p_{i+\omega}}{\sum_{\omega^{\prime}} p_{i+\omega^{\prime}}}
$$

where

$$
p_{i+\omega}=\sigma_{\boldsymbol{x}}^{-(\# \omega)}\left|\mathbf{R}_{i+\omega}\right|^{1 / 2} \exp \left\{\frac{1}{2} \boldsymbol{m}_{i+\omega}^{\mathrm{t}} \mathbf{R}_{i+\omega}^{-1} \boldsymbol{m}_{i+\omega}\right\}\left(\frac{\lambda}{1-\lambda}\right)^{\# \omega}
$$

Compared to a direct implementation using (7)-(9), several sources of computational saving can be found. The main one exploits the fact that both $\mathbf{H}$ and $\mathbf{H}^{\mathrm{t}} \mathbf{H}$ are Toeplitz matrix in the "zero boundary" case of finite convolution. As a consequence, neither $\mathbf{H}_{i+\omega}$, nor $\mathbf{H}_{i+\omega}^{\mathrm{t}} \mathbf{H}_{i+\omega}$, nor $\mathbf{R}_{i+\omega}$ depend on the position $i$. A shorter notation where $i+\omega$ is replaced by $\omega$ will thus be adopted. More importantly, it becomes possible to precompute and store $\mathbf{R}_{\omega}^{-1}$ and $\left|\mathbf{R}_{\omega}\right|$ for all values of $\omega$. An even more efficient scheme manipulates Cholesky factors as detailed in Table $\left[\right.$, such that $\mathbf{U}_{\omega}^{\mathrm{t}} \mathbf{U}_{\omega}=\mathbf{S}_{\omega}, \mathbf{U}_{\omega}$ being an upper-triangular matrix.

Table 6

Implementation of $K$-tuple sampling, Step 1(a),(b) in Table 4.

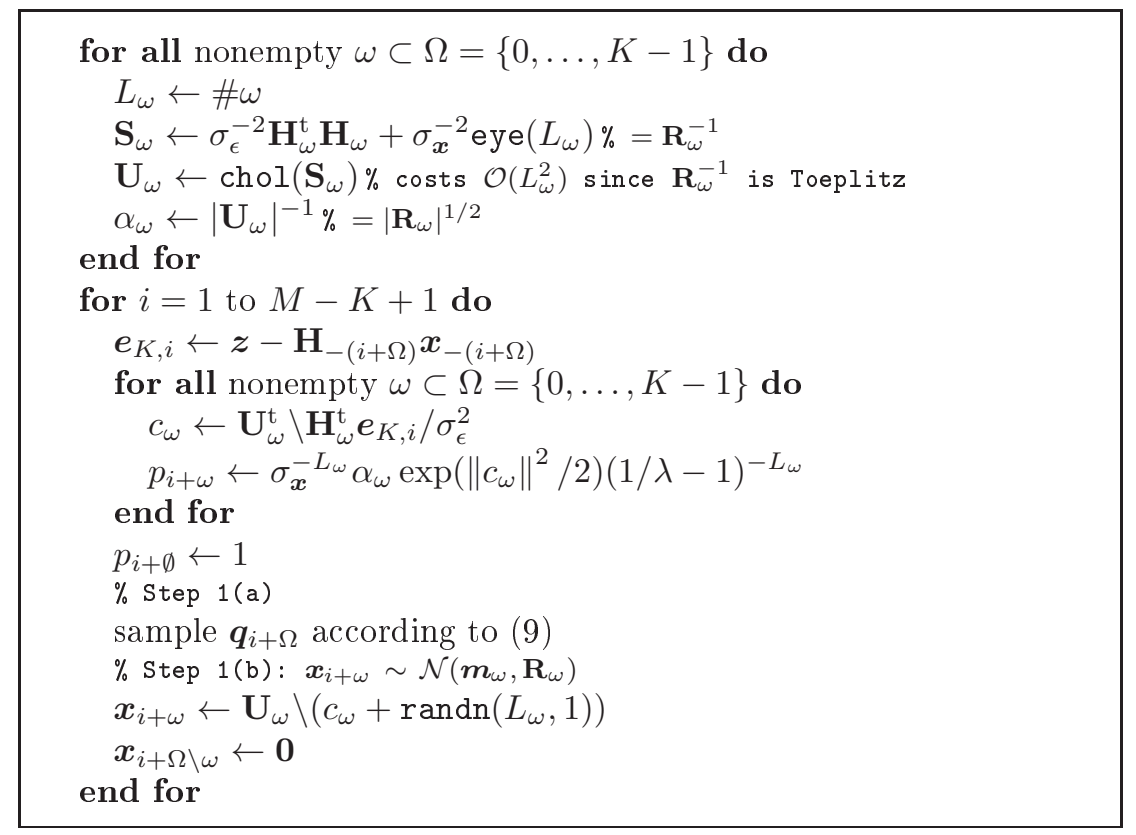

Using an implementation of Table 6 , the iteration numbers of Table 5 can be converted in computer time. This confirms that despite the larger time taken per iteration, the K-tuple sampler may be faster for $K>1$ 
than for $K=1$. However, because of the exponentially increasing cost per iteration as a function of $K$, the best trade-off cannot be reached for large values of $K$. Here and after, we have limited our study to values of $K$ not larger than 4 . Some critical results of the deconvolution problem of the Mendel's sequence in Fig. 2 are reported in Tab. 7 including the overall computational costs using 4 samplers in the $K$-tuple family and the corresponding number of iterations until convergence. More complete simulation results are provided in Section 5

Table 7

Convergence rate of the K-tuple $(K=1, \ldots, 4)$ Gibbs scheme for the example in Fig. 2 measured in iteration numbers and time in seconds. Iteration numbers are noted in each case, e.g. 4600 for the simple hybrid sampler and 900 for the 2 -tuple, etc.

\begin{tabular}{|l||c|c|c|c|}
\hline & $K=1$ & $K=2$ & $K=3$ & $K=4$ \\
\hline Number of iterations & 4600 & 900 & 700 & 600 \\
\hline Computation time (s) & 915 & 202 & 285 & 452 \\
\hline
\end{tabular}

\section{Partially marginal Gibbs sampler}

Here, another sampling scheme is proposed and studied, that indirectly tackles the same inefficiency by marginalizing out $\boldsymbol{x}$ in the step that samples $\boldsymbol{q}$.

\subsection{Partial marginalization of $\boldsymbol{x}$}

In principle, one alternative to the approach in Eq. (44) could be to generate a Markov chain on $\widetilde{\Theta}=$ $\left(\boldsymbol{q}, \boldsymbol{h}, \sigma_{\epsilon}^{2}, \sigma_{h}^{2}, \lambda\right)$ marginalizing $\boldsymbol{x}$, and then build the estimates in two steps: (1) the marginal maximum a posteriori estimate on each binary parameter $q_{i}$ and the posterior mean estimate on the continuous parameters $\widetilde{\Theta} \backslash \boldsymbol{q},(2)$ the posterior mean estimate of $\boldsymbol{x}$ conditional on $(\widetilde{\Theta}, \boldsymbol{z})$, that is a linear estimation problem. Furthermore, a Gibbs sampler with target distribution $P(\widetilde{\Theta} \mid \boldsymbol{z})$ is likely to be more efficient than the hybrid sampler, particularly with respect to the sampling of $\boldsymbol{q}$. Theoretical foundations are available in [13] and [5, Chapter 6.7] regarding the convergence rate of the so called collapsed Gibbs sampler, with the marginalized equilibrium distribution $P(\widetilde{\Theta} \mid \boldsymbol{z})=\int P(\Theta \mid \boldsymbol{z}) d \boldsymbol{x}$. According to [5, Chapter 6.7], the collapsed Gibbs sampler produces a substantial gain in terms of convergence rate if the marginalized parameters form highly dependent pairs with other parameters of the Markov chain (see also [14 and references therein). This is exactly the case of the pair $\{\boldsymbol{x}, \boldsymbol{q}\}$ in the spike train deconvolution problem. Park et al. [15] also illustrates through three examples that such a sampler must be implemented with care to be sure that the desired stationary distribution is preserved. 
However, marginalizing $\boldsymbol{x}$ from the posterior distribution by $P(\widetilde{\Theta} \mid \boldsymbol{z})=\int P(\Theta \mid \boldsymbol{z}) d \boldsymbol{x}$ leads to practical difficulties. Technically, the marginalization of $\boldsymbol{x}$ is only feasible on conditional laws of $\left(\boldsymbol{q}, \lambda, \sigma_{\boldsymbol{h}}^{2}\right)$. On the contrary, the conditional sampling of $\boldsymbol{h}$ becomes extremely difficult if $\boldsymbol{x}$ is integrated out, since $P\left(\boldsymbol{h} \mid \boldsymbol{q}, \sigma_{\boldsymbol{h}}^{2}, \sigma_{\epsilon}^{2}, \boldsymbol{z}\right)$ is a multivariate, non Gaussian law with a complex structure. The same observation is made on the sampling of $\sigma_{\epsilon}^{2}$ if $x$ is marginalized out. Instead of a plain collapsed Gibbs sampler on $\widetilde{\Theta}$, the sampling scheme in Table 8 is proposed to circumvent the direct conditional sampling of $\boldsymbol{h}$ and $\sigma_{\epsilon}^{2}$. Compared with the hybrid sampler, the main difference appears in Step 1, where $\boldsymbol{x}$ has been analytically integrated out. Subsection 4.2 provides an efficient way to implement Step 1.

Table 8

The proposed partially marginal sampler.

\begin{tabular}{l} 
(1) (a) for each $i=1 \ldots, M$, \\
draw $q_{i}^{(k+1 / 2)} \mid \boldsymbol{q}_{1: i-1}^{(k+2)}, \boldsymbol{q}_{i+1: M}^{(k)}, \boldsymbol{h}^{(k)}, \sigma_{\epsilon}^{(k)}, \lambda^{(k)}, \boldsymbol{z}$ \\
(b) draw $\boldsymbol{x}^{(k+1 / 2)} \mid \boldsymbol{q}^{(k+1 / 2)}, \boldsymbol{h}^{(k)}, \sigma_{\epsilon}^{(k)}, \boldsymbol{z}$ \\
(2) (a) draw $\boldsymbol{y}^{(k+1)}$ according to Table 3 \\
(b) draw $\boldsymbol{h}^{(k+1)} \mid \boldsymbol{x}^{(k+1)}, \sigma_{\boldsymbol{h}}^{(k)}, \sigma_{\epsilon}^{(k)}, \boldsymbol{z}$ \\
(3) draw $\sigma_{\epsilon}^{(k+1)} \mid \boldsymbol{x}^{(k+1)}, \boldsymbol{h}^{(k+1)}, \boldsymbol{z}$ \\
(4) draw $\lambda^{(k+1)} \mid \boldsymbol{q}^{(k+1)}$ \\
(5) draw $\sigma_{\boldsymbol{h}}^{(k+1)} \mid \boldsymbol{h}^{(k+1)}$ \\
\hline
\end{tabular}

We give here a mathematical justification that the proposed partially marginal sampler identifies with a Gibbs sampler of the joint posterior law (Eq. (5), that includes $x$ ), for a particular scanning scheme. The techniques are similar to those denoted as marginalization and trimming in [15].

Let $\left(q_{1}, \boldsymbol{x}\right),\left(q_{2}, \boldsymbol{x}\right), \ldots,\left(q_{M}, \boldsymbol{x}\right), \boldsymbol{h}, \sigma_{\epsilon}^{2}, \lambda, \sigma_{\boldsymbol{h}}^{2}$ be the scanning sequence of the Gibbs sampler. For the sampling of couples $\left(q_{i}, \boldsymbol{x}\right)$, a two-stage procedure is considered by applying the Bayes rule $P\left(\boldsymbol{x}, q_{i} \mid \Theta \backslash\left\{\boldsymbol{x}, q_{i}\right\}, \boldsymbol{z}\right)=$ $P\left(q_{i} \mid \Theta \backslash\left\{\boldsymbol{x}, q_{i}\right\}, \boldsymbol{z}\right) P(\boldsymbol{x} \mid \Theta \backslash \boldsymbol{x}, \boldsymbol{z}):$

- draw $q_{i}$ according to $P\left(q_{i} \mid \boldsymbol{q}_{1: i-1}, \boldsymbol{q}_{i+1: M}, \boldsymbol{h}, \sigma_{\epsilon}^{2}, \lambda, \boldsymbol{z}\right)$;

- draw $\boldsymbol{x}$ according to $P\left(\boldsymbol{x} \mid \boldsymbol{q}, \boldsymbol{h}, \sigma_{\epsilon}^{2}, \lambda, \boldsymbol{z}\right)$.

The second stage is therefore repeated $M$ times within each iteration of the sampler, while all but the last one are useless since the correspondingly sampled values of $\boldsymbol{x}$ do not enter any subsequent operation. The $M-1$ corresponding sampling operations can thus be skipped and the resulting sequence of operations coincides exactly with the partially marginal sampler of Table 8, Another mathematical proof is given in [10] by considering Table 8 as a collapsed Gibbs sampler with target distribution $P(\widetilde{\Theta} \mid \boldsymbol{z})$ and then checking the 
invariant condition of the related Markov chain.

In the same conditions than that of Table 5 the partially marginal sampler takes about 20 iterations. Figure 3 illustrates the way it escapes from a local optimal configuration within an acceptable number of iterations in the example of Figure 1. The true configuration is reached after 20 iterations. Moreover, it is observed from the configurations obtained at the 18th and 19th iterations that our scheme is able to radically modify $\boldsymbol{x}$ in one single step, a characteristic directly related to the marginalization of $\boldsymbol{x}$ in Step 1(a) of the sampler.
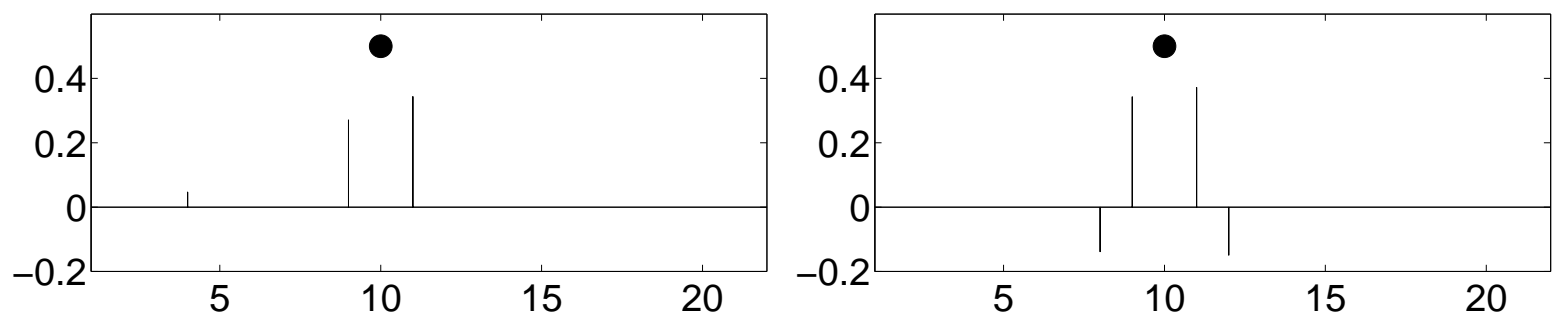

(a) 1st iteration

(b) 18th iteration
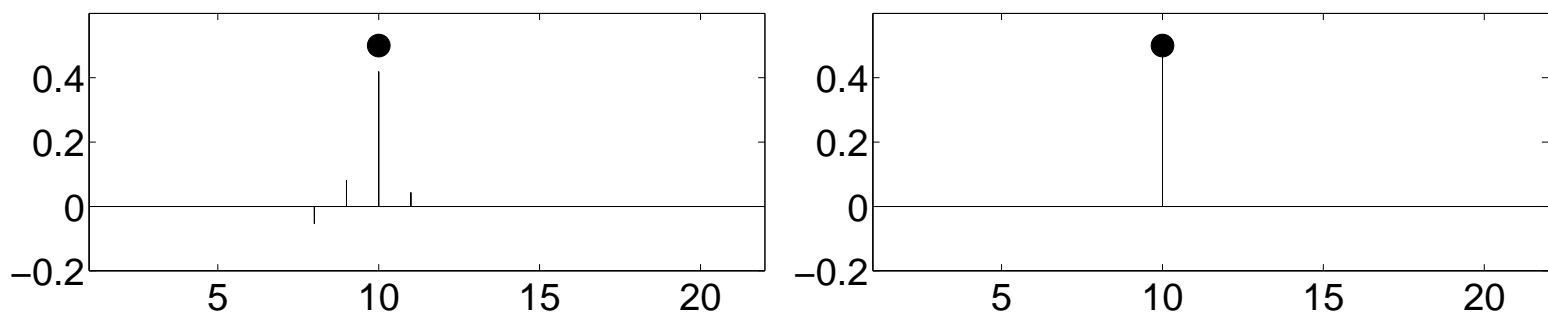

(c) 19 th iteration

(d) 20 th iteration

Fig. 3. Sampled BG sequences obtained by our partially marginal scheme in the example of Figure 1 The Markov chain escapes rapidly the initial configuration neighborhood.

\subsection{Marginal posterior distribution}

In this subsection, a numerical implementation of Step 1 of Table 8 is proposed, inspiring from the recursive method in [4] to evaluate $P\left(q_{i}=0,1 \mid \widetilde{\Theta} \backslash q_{i} ; \boldsymbol{z}\right)$ sequentially. While the latter is based on the storage and update of an $L \times L\left(L=\sum_{i} q_{i}\right)$ matrix, we introduce an even less burdensome strategy by handling the Cholesky factor matrix. The issue of the complexity per iteration is dealt here, whereas the overall computational costs are compared in Section 5 using simulation tests.

First, the conditional posterior distribution of $q_{i}$ takes the following form, as detailed in [4]:

$$
P\left(q_{i} \mid \widetilde{\Theta} \backslash q_{i} ; \boldsymbol{z}\right) \propto|\mathbf{B}|^{-1 / 2} \exp \left(-\frac{1}{2} \boldsymbol{z}^{\mathrm{t}} \mathbf{B}^{-1} \boldsymbol{z}\right)\left(\frac{\lambda}{1-\lambda}\right)^{q_{i}} \propto \exp \left(-f\left(q_{i}\right) / 2\right)
$$


where

$$
\begin{aligned}
\mathbf{B} & =\mathbf{H} \operatorname{diag}(\boldsymbol{q}) \mathbf{H}^{\mathrm{t}}+\sigma_{\epsilon}^{2} \mathbf{I}_{N}, \\
f\left(q_{i}\right) & =\boldsymbol{z}^{\mathrm{t}} \mathbf{B}^{-1} \boldsymbol{z}+\log |\mathbf{B}|+2 q_{i} \log (1 / \lambda-1) .
\end{aligned}
$$

After normalization, the marginal probability of $q_{i}$ reads:

$$
P\left(q_{i} \mid \widetilde{\Theta} \backslash q_{i}, \boldsymbol{z}\right)=\left(1+\exp \left(-\left(f\left(1-q_{i}\right)-f\left(q_{i}\right)\right) / 2\right)\right)^{-1},
$$

which reduces to the evaluation of $f\left(1-q_{i}\right)-f\left(q_{i}\right)$, using the following steps [4]:

$$
\begin{aligned}
\tau_{i} & =\delta_{i}+\sigma_{\epsilon}^{-2} \boldsymbol{h}_{i}^{\mathrm{t}} \widetilde{\mathbf{B}}_{0}^{-1} \boldsymbol{h}_{i} \\
\widetilde{\mathbf{B}}_{i}^{-1} & =\widetilde{\mathbf{B}}_{0}^{-1}-\sigma_{\epsilon}^{-2} \widetilde{\mathbf{B}}_{0}^{-1} \boldsymbol{h}_{i} \tau_{i}^{-1} \boldsymbol{h}_{i}^{\mathrm{t}} \widetilde{\mathbf{B}}_{0}^{-1} \\
\left|\widetilde{\mathbf{B}}_{i}^{-1}\right| & =\left|\widetilde{\mathbf{B}}_{0}^{-1}\right| \delta_{i} \tau_{i}
\end{aligned}
$$

with $\widetilde{\mathbf{B}}=\mathbf{B} / \sigma_{\epsilon}^{2}$. $\delta_{i}= \pm 1$ depends on whether 1 is added or removed at $q_{i}$ and $\widetilde{\mathbf{B}}_{i}$ and $\widetilde{\mathbf{B}}_{0}$ differ only at $q_{i}$. Let us note that $\widetilde{\mathbf{B}}_{0}, \widetilde{\mathbf{B}}_{i}>0$, so that it can be deduced from (14) that $\tau_{i}$ has the same sign as $\delta_{i}$.

Further simplifications are also introduced in [4] by exploiting the sparse nature of $\widetilde{\mathbf{B}}$ and applying the matrix inversion lemma. Noticing that the rank of $\mathbf{H} \operatorname{diag}(\boldsymbol{q}) \mathbf{H}^{\mathrm{t}}$ is only $L, \widetilde{\mathbf{B}}$ takes the alternate form $\widetilde{\mathbf{B}}=$ $\sigma_{\epsilon}^{-2} \mathbf{G G}^{\mathrm{t}}+\mathbf{I}$, where $\mathbf{G}=\mathbf{H D}$ is full rank, and $\mathbf{D}$ made of the nonzero columns of $\operatorname{diag}(\boldsymbol{q})$. By applying the matrix inversion lemma, we have

$$
\widetilde{\mathbf{B}}^{-1}=\mathbf{I}-\sigma_{\epsilon}^{-2} \mathbf{G C}^{-1} \mathbf{G}^{\mathrm{t}}
$$

where $\mathbf{C}=\sigma_{\epsilon}^{-2} \mathbf{G}^{\mathrm{t}} \mathbf{G}+\mathbf{I}$ is a $L \times L$ matrix. Therefore, $\mathbf{C}^{-1}(L \times L)$ can be stored and updated instead of $\widetilde{\mathbf{B}}^{-1}$ $(N \times N)$. In the case of adding a spike at $q_{i}$, the formula for the update of $\mathbf{C}_{i}^{-1}$ up to a matrix permutation operation is the following:

$$
\begin{aligned}
\boldsymbol{b} & =-\sigma_{\epsilon}^{-2} \tau_{i}^{-1} \mathbf{C}_{0}^{-1} \mathbf{G}_{0}^{\mathrm{t}} \boldsymbol{h}_{i} \\
\mathbf{C}_{i}^{-1} & =\left[\begin{array}{cc}
\mathbf{C}_{0}^{-1}+\boldsymbol{b} \tau_{i} \boldsymbol{b}^{\mathrm{t}} & \boldsymbol{b} \\
\boldsymbol{b}^{\mathrm{t}} & \tau_{i}^{-1}
\end{array}\right]
\end{aligned}
$$

The case of removing a spike is straightforward since we have the following: 


$$
\mathbf{C}_{0}^{-1}=\left[\begin{array}{cc}
\mathbf{C}_{i}^{-1}+\boldsymbol{b} \tau_{i} \boldsymbol{b}^{\mathrm{t}} & \boldsymbol{b} \\
\boldsymbol{b}^{\mathrm{t}} & \tau_{i}^{-1}
\end{array}\right]
$$

Notice that in the case of spike removal, $\boldsymbol{b}$ and $\tau_{i}$ are extracted from $\mathbf{C}_{0}^{-1}$ rather than calculated as in Eq. (16). These operations are invariant up to a matrix permutation in cases of adding/removing a spike at an arbitrary location.

Finally, we propose to further reduce the computation and memory load, by updating and storing the Cholesky factor $\mathbf{F}$ of $\mathbf{C}^{-1}\left(\mathbf{F}^{\mathrm{t}} \mathbf{F}=\mathbf{C}^{-1}\right.$ for an upper-triangular matrix $\left.\mathbf{F}\right)$. It is derived from Equations (13) and (15) that:

$$
\begin{aligned}
\tau_{i} & =\delta_{i}+\sigma_{\epsilon}^{-2}\left\|\boldsymbol{h}_{i}\right\|^{2}-\sigma_{\epsilon}^{-4}\left\|\mathbf{F}_{0} \mathbf{G}_{0}^{\mathrm{t}} \boldsymbol{h}_{i}\right\|^{2}, \\
\boldsymbol{z}^{\mathrm{t}} \widetilde{\mathbf{B}}_{0}^{-1} \boldsymbol{h}_{i} & =\boldsymbol{z}^{\mathrm{t}} \boldsymbol{h}_{i}-\sigma_{\epsilon}^{-2}\left(\mathbf{F}_{0} \mathbf{G}_{0}^{\mathrm{t}} \boldsymbol{z}\right)^{\mathrm{t}}\left(\mathbf{F}_{0} \mathbf{G}_{0}^{\mathrm{t}} \boldsymbol{h}_{i}\right)
\end{aligned}
$$

and from Eq. (11) and (13) that:

$$
f\left(1-q_{i}\right)-f\left(q_{i}\right)=\log \left(\delta_{i} \tau_{i}\right)-\sigma_{\epsilon}^{-4} \tau_{i}^{-1}\left(\boldsymbol{z}^{\mathrm{t}} \widetilde{\mathbf{B}}_{0}^{-1} \boldsymbol{h}_{i}\right)^{2}+2 \delta_{i} \log \left(\frac{1}{\lambda}-1\right)
$$

Two extra advantages of the Cholesky decomposition are:

- since the conditional law in Step 1(b) reads

$$
(\boldsymbol{x} \mid \widetilde{\boldsymbol{\Theta}}, \boldsymbol{z}) \sim \mathcal{N}\left(\sigma_{\epsilon}^{-2} \mathbf{C}^{-1} \mathbf{G}_{0}^{\mathrm{t}} \boldsymbol{z}, \mathbf{C}^{-1}\right),
$$

sampling $\boldsymbol{x} \operatorname{costs} \mathcal{O}\left(L^{2}\right)$ using $\mathbf{F}$ (See Table 9);

- the incremental update of $\mathbf{F}$ also costs $\mathcal{O}\left(L^{2}\right)$ using a rank-1 Cholesky update method. The examples of adding and removing a spike at the last location is given in the following for notational simplicity. It is however detailed in the appendix that unlike adding a spike at at an arbitrary location, the case of removing a spike at an arbitrary location requires two operations of Cholesky rank-1 update.

For adding a spike at the last location, $\tau_{i}>0$ and equation (17) also reads:

$$
\mathbf{C}_{i}^{-1}=\left[\begin{array}{ll}
\mathbf{F}_{0}^{\mathrm{t}} & \mathbf{0} \\
\mathbf{0}^{\mathrm{t}} & 0
\end{array}\right]\left[\begin{array}{ll}
\mathbf{F}_{0} & \mathbf{0} \\
\mathbf{0}^{\mathrm{t}} & 0
\end{array}\right]+\left[\begin{array}{c}
\boldsymbol{b} \tau_{i}^{1 / 2} \\
\tau_{i}^{-1 / 2}
\end{array}\right]\left[\begin{array}{c}
\boldsymbol{b} \tau_{i}^{1 / 2} \\
\tau_{i}^{-1 / 2}
\end{array}\right]^{\mathrm{t}}
$$

In the case of removing a spike at the last location, from Eq. (18) and the definition of Cholesky decomposition, we have: 


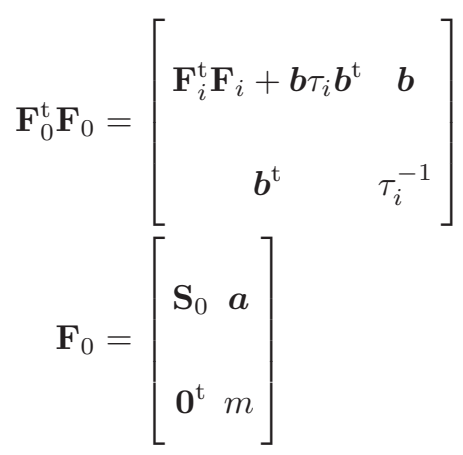

where $\mathbf{S}_{0}$ is also an upper triangular matrix. It is then directly deduced that:

$$
\begin{aligned}
\mathbf{S}_{0}^{\mathrm{t}} \boldsymbol{a} & =\boldsymbol{b} \\
\boldsymbol{a}^{\mathrm{t}} \boldsymbol{a}+m^{2} & =\tau_{i}^{-1} \\
\mathbf{S}_{0}^{\mathrm{t}} \mathbf{S}_{0} & =\mathbf{F}_{i}^{\mathrm{t}} \mathbf{F}_{i}+\boldsymbol{b} \tau_{i} \boldsymbol{b}^{\mathrm{t}}
\end{aligned}
$$

Let cholupdate $\left(\mathbf{A}, \boldsymbol{d},{ }^{\prime} \pm^{\prime}\right)$ be the method that updates in $\mathcal{O}\left(L^{2}\right)$ the Cholesky factor of $\mathbf{A}^{\mathrm{t}} \mathbf{A} \pm \boldsymbol{d} \boldsymbol{d}^{\mathrm{t}}$, where $\mathbf{A}$ is already an upper triangular matrix. Then, $\mathbf{F}_{i}=$ cholupdate $\left(\mathbf{S}_{0}, \boldsymbol{b} \tau_{i}^{1 / 2},^{\prime}-^{\prime}\right)$.

\section{Simulation results}

\subsection{Convergence diagnostic}

In order to compare empirically the convergence speed of the different samplers, we have resorted to Brooks and Gelman's iterated graphical method to assess convergence [12. This diagnostic method is based upon the covariance estimation of $m$ independent Markov chains $\left\{\Phi_{j t}, j=1, \ldots, m ; t=1, \ldots, n\right\}$ of equal length $n$. Let $\bar{\Phi}_{j}$. (respectively, $\bar{\Phi}_{. .}$) denote the local (respectively, global) mean of the chains. The intra-chain and inter-chain variances are defined as covariance matrix averages:

$$
\begin{aligned}
& \mathbf{V}_{\text {intra }}=\frac{1}{m(n-1)} \sum_{j=1}^{m} \sum_{t=1}^{n}\left(\Phi_{j t}-\bar{\Phi}_{j .}\right)\left(\Phi_{j t}-\bar{\Phi}_{j .}\right)^{\mathrm{t}} \\
& \mathbf{V}_{\text {inter }}=\frac{1}{m-1} \sum_{j=1}^{m}\left(\bar{\Phi}_{j .}-\bar{\Phi}_{. .}\right)\left(\bar{\Phi}_{j .}-\bar{\Phi}_{. .}\right)^{\mathrm{t}}
\end{aligned}
$$

that characterize the convergence behavior. Brooks and Gelman [12] proposed to evaluate the multivariate potential scale reduction factor (MPSRF):

$$
\operatorname{MPSRF}=\frac{n-1}{n}+\frac{m+1}{m} \lambda\left(\mathbf{V}_{\text {intra }}^{-1} \mathbf{V}_{\text {inter }}\right)
$$


Table 9

Step 1 of the partially marginal sampling scheme. The update of $\mathbf{F}$ is given here up to a matrix permutation.

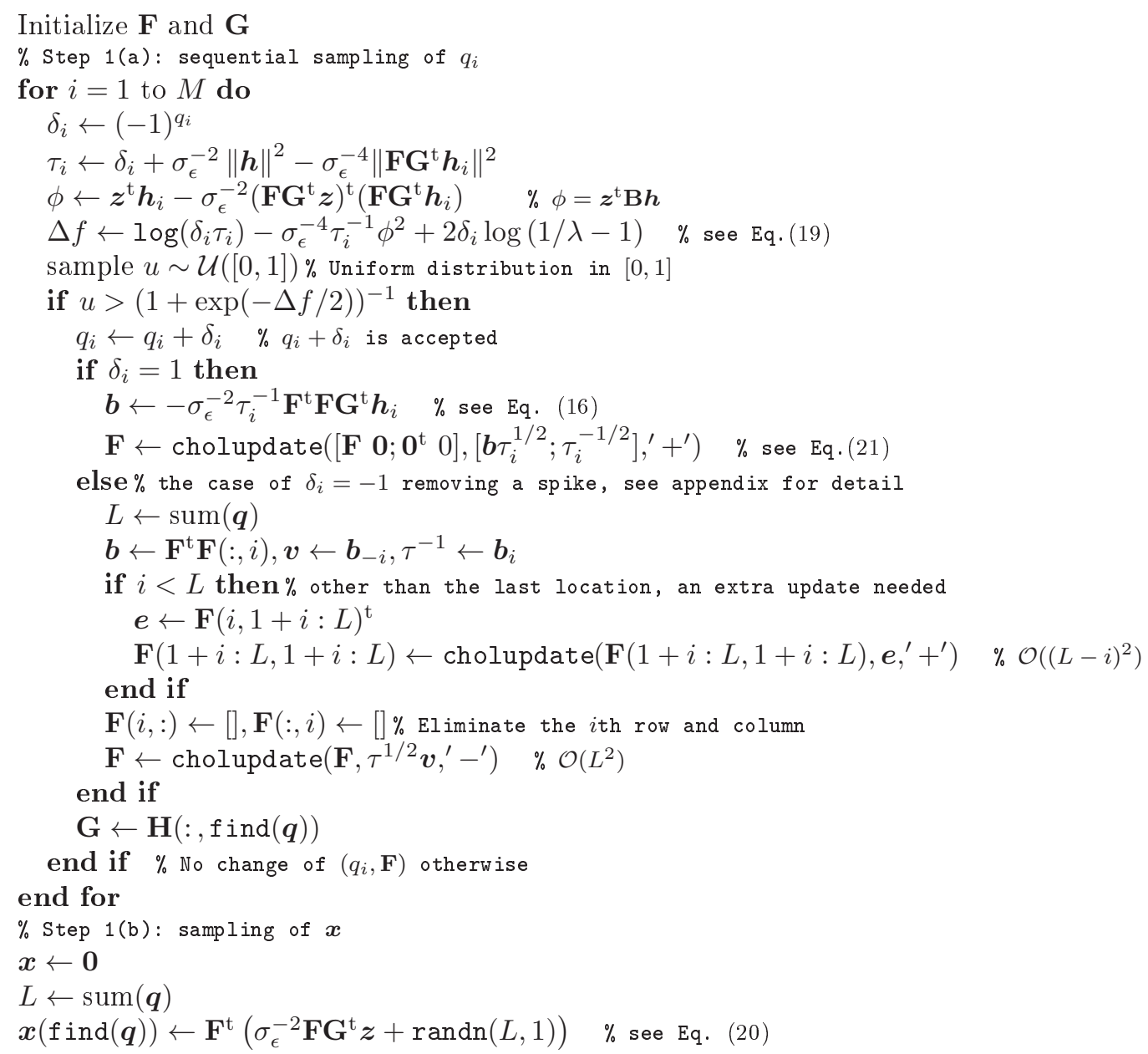

where $\lambda(\cdot)$ returns the largest eigenvalue of the covariance matrix. Convergence is diagnosed when MPSRF is close to one (e.g., MPSRF < 1.2 as proposed in [12]). In order to get a graphical evolution of the convergence, each chain is divided into batches of $b$ samples, and the MPSRF is calculated upon the second halves of the Markov chains $\left\{\Phi_{j t}\right\}, t=1, \ldots, k b$ of increasing lengths $k b$, while the first halves are regarded as a burn-in period. From an empirical basis, it is also advised to select $b \approx n / 20$ in [12].

\subsection{Simulation tests}

A test scenario is designed here to compare the generalized K-tuple Gibbs sampler (including the classical hybrid sampler in the case $K=1$ ) of Section 3 and the partially marginal sampler of Section 4 in terms of robustness with respect to different random initial conditions. Time-shift and scale ambiguities are taken 
into account in all the methods, as described in Section 2.2. Let us reexamine the example of the benchmark sequence (Mendel's sequence, for which $M=300$ and $\lambda=0.1$.) in Figure 2. To concentrate on the convergence quality of the Bernoulli sequence, we evaluate the MPSRF evolutions of $\left\{\boldsymbol{q}^{(k)}\right\}$ for ten independent Markov chains, i.e., $m=10$. Figure 4 is compares the convergence rate of $K$-tuple samplers for different values of $K$ : while MPSRF falls under 1.2 after 515 seconds of simulation for the classical hybrid sampler $(K=1)$, this threshold is reached after about 285 seconds for the 3-tuple sampler and in less than 202 seconds for the 2-tuple sampler. It is interesting to observe that both the 3 and 4 -tuple sampler have a more stable convergence behavior in the convergence zone while the hybrid sampler presents the most oscillating MPSRF values. These results confirm the discussion in Section 3, augmenting $K$ improves the convergence quality in terms of iteration numbers while in the meantime increases the computational load per iteration. The overall performance relies on the trade-off between the two criteria. Thus, the best compromise in the $K$-tuple sampler series is reached for $K=2$ in the given example.

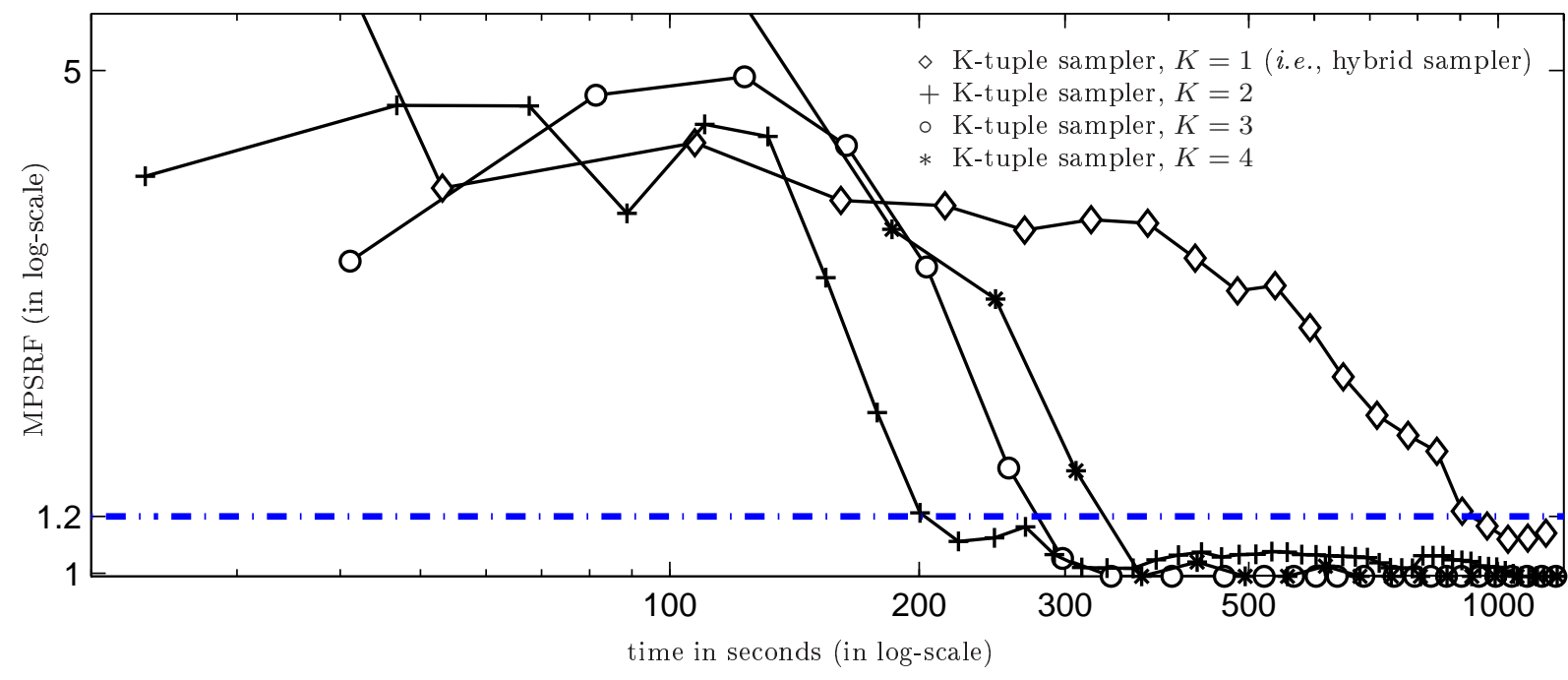

Fig. 4. Evolution of MPSRF for the $K$-tuple sampler family $(K=1,2,3)$ in the case of Mendel's sequence (SNR $=12.80 \mathrm{~dB})$.

Next we compare the convergence diagnostic results on the 2-tuple sampler and the partially marginal sampler. Each MPSRF in Figure 5 is evaluated for every $b=100$ iterations. Altogether 300 iterations (116 seconds of simulation) are required for the partially marginalized sampler to reach convergence, not to mention the overall lower level of MPSRF in the convergence zone compared with the 2 -tuple sampler. Thus the partially marginalized sampler takes fewer iterations and less time to converge than the best selected $K$-tuple sampler in Figure 4. We also point out that the asymptotic complexity per iteration of the partially marginal sampler is lower than that of the 2-tuple sampler in the given simulation example, though the 
contrary is observed for the first 100 samples (corresponding to the heating period) in which the number of detected spikes $L$ is relatively important.

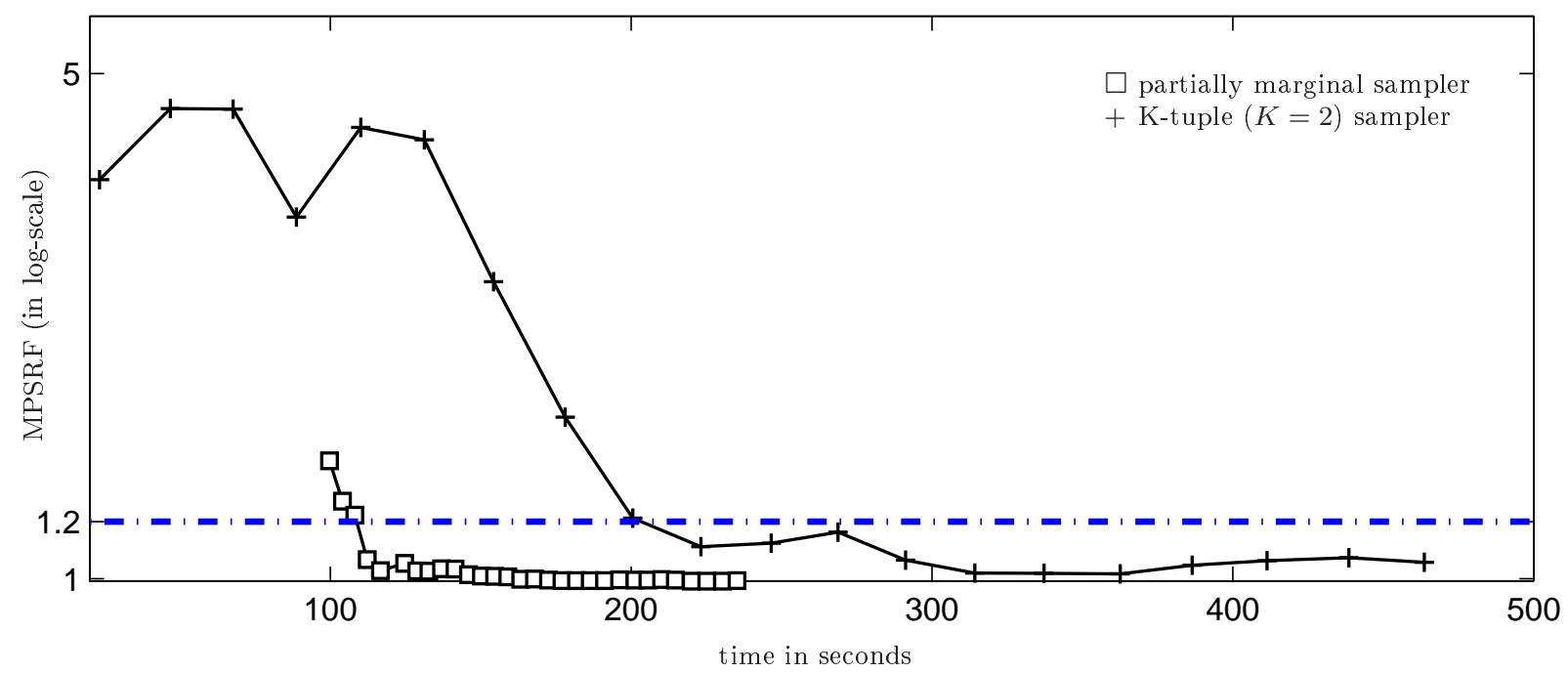

Fig. 5. Evolution of MPSRF for the partially marginal sampler and the 2-tuple sampler in the case of Mendel's sequence. MPSRF has been computed every 100 samples for both methods.

To further illustrate its robustness, deconvolution results obtained by the partially marginal sampler are shown in Figure 6 under identical initial conditions as in Figure 2, Only one of the results is reported, as they are undistinguishable from each other (perfectly consistent estimations). And this is true for all the samplers once their MPSRF values drop below the 1.2 threshold.
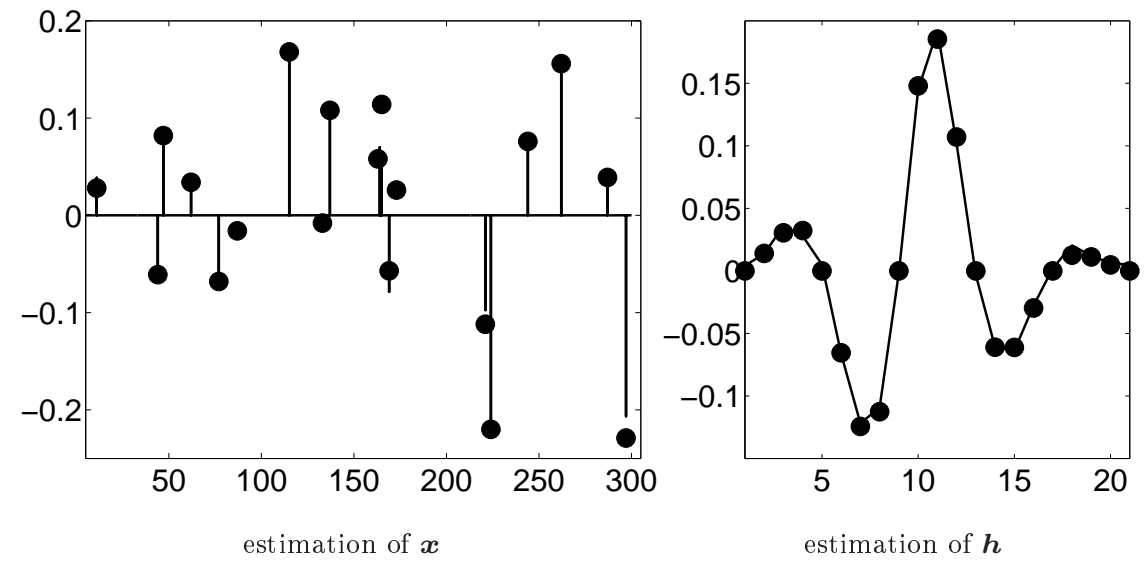

Fig. 6. Estimation result using the proposed sampler, under the same conditions as in Figure 2. The 10 independent Markov chains yield undistinguishable estimates.

In the given example of the Mendel's sequence (for which $M=300$ ), the partially marginal sampler achieves a better compromise between the two criteria [5] than the most performant sampler in the generalized $K$-tuple family: the time required to reach convergence is reduced by half. However, the partially 
marginal sampler do not necessarily outperform the $K$-tuple sampler in dealing with longer datasets. Notice that the computational complexity for a Cholesky update operation in the latter case is $\mathcal{O}\left(L^{2}\right)\left(L=\sum q_{i} \approx\right.$ $\lambda M$, the number of detected spikes), thus proportional to the data length of the BG sequence, whereas the complexity per iteration of the $K$-tuple sampler is non-increasing with respect to the number of detected spikes $L$. To illustrate the disadvantage of the partially marginal sampler, it is necessary to test on simulation examples of longer observation data $z$ while fixing the Bernoulli parameter $\lambda$. Figure 7 compares the complexity per iteration as a function of BG sequence length $M$ for the two algorithms. The 2-tuple sampler potentially outperforms the partially marginal sampler due to its linear growth of complexity per iteration with respect to $M$. Figure 8 resumes the convergence time for all the 5 samplers $(K=1, \ldots, 4$ in solide lines and the partially marginalised sampler in dashed line) on simulation data whose lengths vary from 100 to 1600 and $\lambda=10 \%$. While the partially marginal sampler outperforms all the rest algorithms in areas of shorter data length $(M<800)$, its quadratic computation cost per iteration as shown in Fig. 7 yields the contrary in applications with longer data lengths. We also note that for the given bernoulli parameter $\lambda=10 \%$, the hybrid sampler is never a good choice inside the $K$-tuple family regardless of the data length.

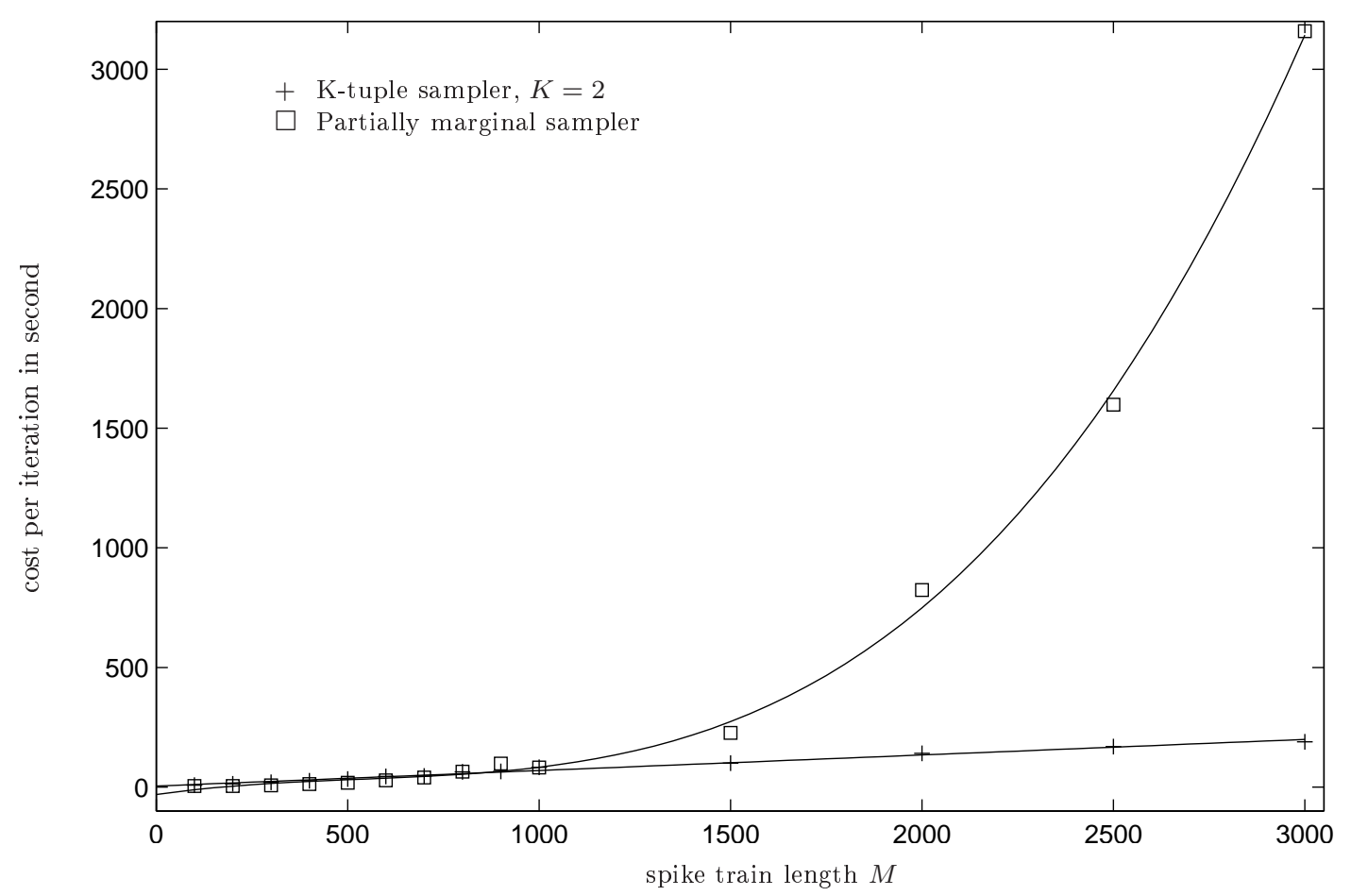

Fig. 7. Computation cost per iteration comparison between the 2-tuple Gibbs sampler and the partially marginal sampler. The two methods are tested on the same simulated observation data $\boldsymbol{z}$, for which the length of the spike train ranges from 100 to 3000 and the Bernoulli parameter $\lambda$ is fixed to $10 \%$. Polynomial interpolations (of degree 1 and 2 respectively) are traced to show the quasi-linear and -quadratic evolution of complexity per iteration with respect to the spike train length $M$. 


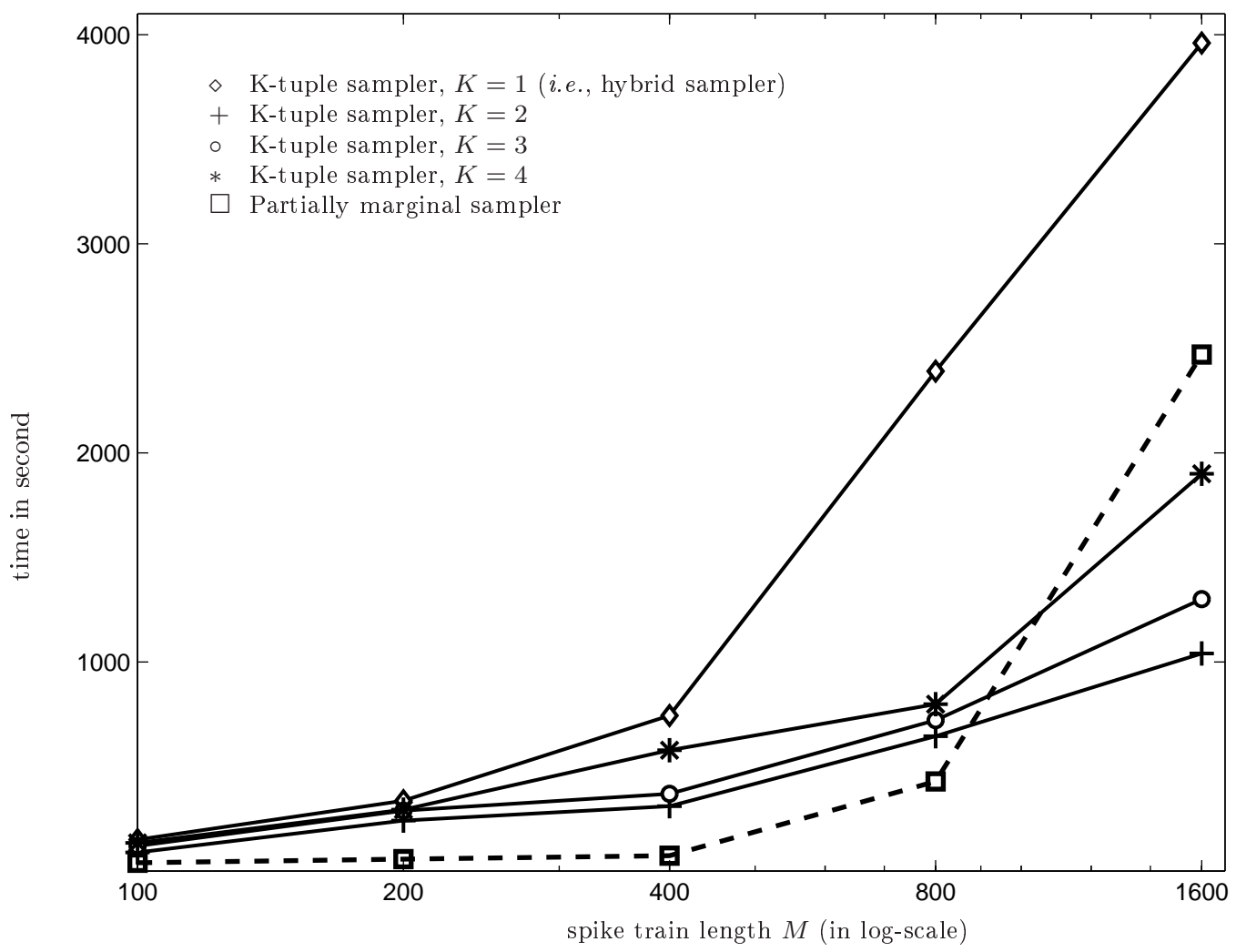

Fig. 8. Convergence time comparison for the 5 samplers applied on spike trains of increasing length.

\section{Conclusion}

This paper proposes two distinct methods in dealing with the identified inefficiency concerning the Bernoulli label $\boldsymbol{q}$ sampling in the BG deconvolution problem. Detailed algorithms are given for both methods as well as their performance on simulation tests. It is shown that both methods are mathematically valid MCMC sampling schemes and both achieve better convergence properties in comparison to the hybrid sampler, the latter is based on Cheng et al.'s Gibbs scheme with time-shift compensations and scale re-sampling. Both proposed methods demonstrate better trade-off for the convergence rate by reducing drastically the iteration steps needed to converge. On the simulation test of Mendel's sequence, the partially marginal sampler is shown to out-perform the whole class of K-tuple samplers, among which the optimized parameter is found at $K=2$. This is however due to the relatively small size of the simulation problem and the contrary is observed by simply augmenting the simulated data length.

We conclude with some extensions of the proposed techniques. By combining the idea of a K-tuple joint sampling and that of partial marginalization of $\boldsymbol{x}$, i.e., updating $\boldsymbol{q}_{i+\Omega} \mid \boldsymbol{q}_{-(i+\Omega)}, \boldsymbol{h}, \sigma_{\epsilon}, \lambda$ in Step 1 of Table 8 , one might further reduce the number of iterations necessary to escape from local optima at the cost of higher 
computation load per iteration. Such a solution should be adopted when the deconvolution problem presents more difficulties, characterized by either low SNR, band-limited IR and/or less sparse spike trains.

APPENDIX To update the Cholesky factor $\mathbf{R}$ on removing a spike at the location $i$ such that $\mathbf{K}^{\mathrm{t}} \mathbf{K}=$ $\mathbf{R}^{\mathrm{t}} \mathbf{R}-\boldsymbol{v} \boldsymbol{v}^{\mathrm{t}}$, we specify :

$$
\begin{array}{r}
\mathbf{R}=\left[\begin{array}{lll}
\mathbf{A} & \boldsymbol{b} & \mathbf{C} \\
0 & \delta & \boldsymbol{e}^{\mathrm{t}}
\end{array}\right], \mathbf{K}=\left[\begin{array}{ccc}
\mathbf{G} & 0 & \mathbf{H} \\
0 & 0 & \mathbf{F}
\end{array}\right] \\
\left\{\begin{array}{lll}
0 & 0 & 0 \\
0 & 0 & \mathbf{J}
\end{array}\right], \\
\tau^{-1}=\boldsymbol{b}^{\mathrm{t}} \boldsymbol{b}+\delta^{2}
\end{array}
$$

where $\{\mathbf{A}, \mathbf{F}, \mathbf{G}, \mathbf{J}\}$ are upper-triangular matrices and $\delta=\mathbf{R}_{i, i}$ the $i$ th diagonal value of $\mathbf{R} .\left(\mathbf{K}_{-i,-i}\right)$ is therefor the updated Cholesky factor.

A most evident solution consists an operation $\mathbf{K}=$ Cholupdate $\left(\mathbf{R}, \boldsymbol{v},{ }^{\prime}-^{\prime}\right)$ followed by an extraction to get the updated Cholesky factor (of lowered dimension) :

$$
\left[\begin{array}{cc}
\mathbf{G} & \mathbf{H} \\
0 & \mathbf{J}
\end{array}\right] \text {, }
$$

which however systematically fails due to the fact that $\mathbf{K}^{t} \mathbf{K}$ is no longer definite positive. In fact, one necessary condition [16] for a successful rank-1 downdate operation (update by subtracting $\boldsymbol{v} \boldsymbol{v}^{\mathrm{t}}$ ) is that $\rho^{2}=1-\boldsymbol{p}^{\mathrm{t}} \boldsymbol{p}>0$ for which $\boldsymbol{p}$ is defined by

$$
\mathbf{R}^{\mathrm{t}} \boldsymbol{p}=\boldsymbol{v}
$$

Without inverting $\mathbf{R}^{\mathrm{t}}$, it can be deduced from (A-2) that $\boldsymbol{p}=\sqrt{\tau} \mathbf{R}(:, j)$ and thus $\rho^{2}=0$. The algorithm of Cholupdate by a series of Givens rotation should break down according to [16].

The problem of non-definite positiveness is avoided by a strategy that first lower the dimension and then perform the Cholupdate algorithm. From (A-1), we obtain: 


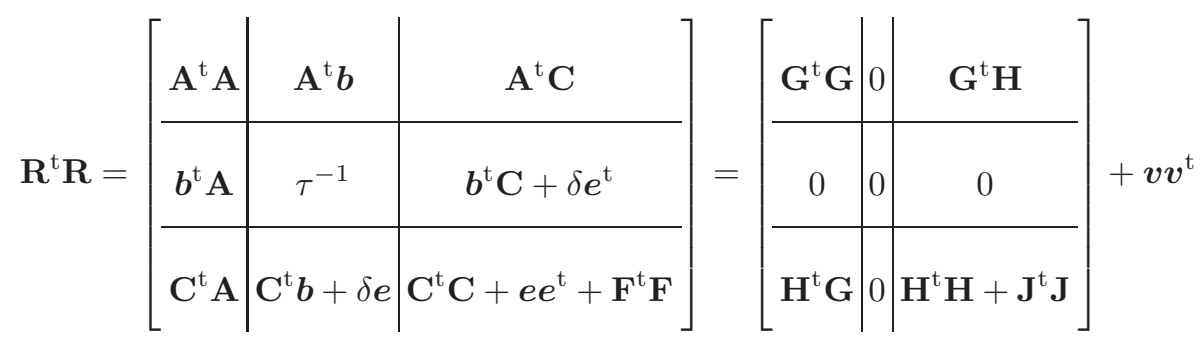

The submatrices on both sides by eliminating the $i$ th row and column yields :

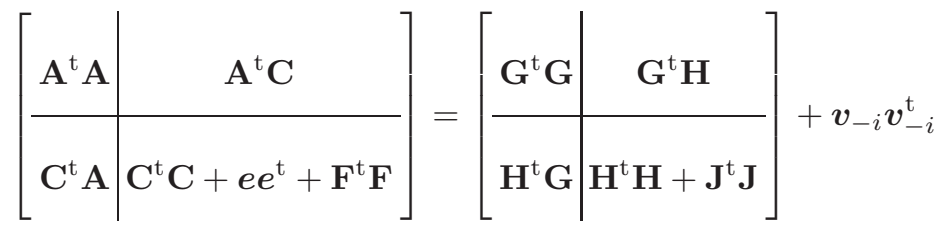

for which $\boldsymbol{v}_{-i}$ denotes $\boldsymbol{v}$ without the $i$ th component. The following relation is key to the algorithm that we propose :

$$
\left[\begin{array}{ll}
\mathbf{A} & \mathbf{C} \\
0 & \mathbf{F}
\end{array}\right]^{\mathrm{t}}\left[\begin{array}{ll}
\mathbf{A} & \mathbf{C} \\
0 & \mathbf{F}
\end{array}\right]+\left[\begin{array}{l}
0 \\
e
\end{array}\right]\left[\begin{array}{l}
0 \\
e
\end{array}\right]^{\mathrm{t}}=\left[\begin{array}{ll}
\mathbf{G} & \mathbf{H} \\
0 & \mathbf{J}
\end{array}\right]^{\mathrm{t}}\left[\begin{array}{cc}
\mathbf{G} & \mathbf{H} \\
0 & \mathbf{J}
\end{array}\right]+\boldsymbol{v}_{-i} \boldsymbol{v}_{-i}^{\mathrm{t}}
$$

The two steps that leads to a spike removing update are :

(i) an update on $\mathbf{F}$ (of reduced dimension):

$$
\mathbf{M}=\text { Cholupdate }\left(\mathbf{F}, e^{\prime}+^{\prime}\right)
$$

(ii) a downdate that keeps the positiveness of the product matrix and will not fail:

$$
\left[\begin{array}{ll}
\mathbf{G} & \mathbf{H} \\
0 & \mathbf{J}
\end{array}\right]=\text { Cholupdate }\left(\left[\begin{array}{ll}
\mathbf{A} & \mathbf{C} \\
0 & \mathbf{M}
\end{array}\right], \boldsymbol{v}_{-i},{ }^{\prime}-^{\prime}\right) \text {. }
$$

it can be proven that $\rho^{2}=1-\boldsymbol{p}^{\mathrm{t}} \boldsymbol{p}$ is strictly positive in this case.

\section{References}

[1] Q. Cheng, R. Chen, and T.-H. Li, "Simultaneous wavelet estimation and deconvolution of reflection seismic signals", IEEE Trans. Geosci. Remote Sensing, vol. 34, pp. 377-384, Mar. 1996. 
[2] J. J. Kormylo and J. M. Mendel, "Maximum-likelihood seismic deconvolution", IEEE Trans. Geosci. Remote Sensing, vol. GE-21, no. 1, pp. 72-82, Jan. 1983.

[3] S. Bourguignon and H. Carfantan, "Bernoulli-Gaussian spectral analysis of unevenly spaced astrophysical data", in IEEE Workshop Stat. Sig. Proc., Bordeaux, France, July 2005, pp. 811-816.

[4] F. Champagnat, Y. Goussard, and J. Idier, "Unsupervised deconvolution of sparse spike trains using stochastic approximation", IEEE Trans. Signal Processing, vol. 44, no. 12, pp. 2988-2998, Dec. 1996.

[5] J. S. Liu, Monte Carlo Strategies in Scientific Computing, Springer Series in Statistics. Springer Verlag, New York, NY, 2001.

[6] C. P. Robert and G. Casella, Monte Carlo Statistical Methods, Springer Texts in Statistics. Springer Verlag, New York, NY, 2nd edition, 2004.

[7] C. Labat and J. Idier, "Sparse blind deconvolution accounting for time-shift ambiguity", in Proc. IEEE ICASSP, Toulouse, France, May 2006, pp. 616-619.

[8] T. Veit, J. Idier, and S. Moussaoui, "Rééchantillonnage de l'échelle dans les algorithmes MCMC pour les problèmes inverses bilinéaires", Traitement du Signal, vol. 25, no. 4, pp. 329-343, 2008.

[9] C. Y. Chi and J. M. Mendel, "Improved maximum-likelihood detection and estimation of Bernoulli-Gaussian processes", IEEE Trans. Inf. Theory, vol. 30, pp. 429-435, 1984.

[10] D. Ge, J. Idier, and E. Le Carpentier, "A new MCMC algorithm for blind Bernoulli-Gaussian deconvolution", in EUSIPCO, Lausanne, Switzerland, Sep. 2008.

[11] N. Dobigeon, J.-Y. Tourneret, and J. D. Scargle, "Joint segmentation of multivariate astronomical times series: Bayesian sampling with a hierarchical model", IEEE Trans. Signal Processing, vol. 55, no. 2, pp. 414-423, Feb. 2007.

[12] S. P. Brooks and A. Gelman, "General methods for monitoring convergence of iterative simulations", J. Comput. Graph. Statist., vol. 7, no. 4, pp. 434-455, 1998.

[13] J. S. Liu, W. H. Wong, and A. Kong, "Covariance structure of the Gibbs sampler with applications to the comparisons of estimators and augmentation schemes", Biometrika, vol. 81, pp. 27-40, 1994.

[14] O. Cappé, A. Doucet, M. Lavielle, and E. Moulines, "Simulation-based methods for blind maximum-likelihood filter identification", Signal Processing, vol. 73, no. 1-2, pp. 3-25, Feb. 1999.

[15] T. Park and D. A. van Dyk, "Partially Collapsed Gibbs Samplers: Illustrations and Applications", Journal of Computational and Graphical Statistics, vol. 18, no. 2, pp. 283-305, 2009.

[16] M. Seeger, "Low Rank Updates for the Cholesky Decomposition", Tech. Rep., Department of EECS, University of California at Berkeley, 485 Soda Hall, Berkeley CA, 2008. 